\title{
BOUNDED DISTRIBUTIVE LATTICE EXPANSIONS
}

\author{
MAI GEHRKE and BJARNI JÓNSSON
}

\begin{abstract}
A new notion of a canonical extension $\mathbf{A}^{\sigma}$ is introduced that applies to arbitrary bounded distributive lattice expansions (DLEs) A. The new definition agrees with the earlier ones whenever they apply. In particular, for a bounded distributive lattice $\mathbf{A}, \mathbf{A}^{\sigma}$ has the same meaning as before.

A novel feature is the introduction of several topologies on the universe of the canonical extension of a DL. One of these topologies is used to define the canonical extension $f^{\sigma}: \mathbf{A}^{\sigma} \rightarrow \mathbf{B}^{\sigma}$ of an arbitrary map $f: \mathbf{A} \rightarrow \mathbf{B}$ between DLs, and hence to define the canonical extension $\mathbf{A}^{\sigma}$ of an arbitrary DLE A. Together the topologies form a powerful tool for showing that many properties of DLEs are preserved by canonical extensions.
\end{abstract}

\section{Introduction}

The usefulness of the notion of canonical extension was demonstrated in [15], [16] and [13]. In [15], and [16] it was shown, among other things, that every relation algebra can be embedded in a complete and atomic relation algebra, and that every closure algebra can be embedded in the complex algebra of a partially ordered set. The principal result in [13] is an algebraic proof of an important theorem from modal logic. However, in light of the present interest in lattice ordered algebras and in non-standard propositional logics, the class of algebras to which the original notion of canonical extensions applies is too narrow. In [8], a modest step was taken towards correcting this by dropping the requirement of complementation, i.e., by considering distributive lattices with operators. A much larger step was taken in [9], where the auxiliary operations were not required to be operators. In fact, the only requirement was that, as a function of any one of its arguments, each function be either isotone or antitone. We now drop even this condition and consider algebras $\mathbf{A}=\left(\mathbf{A}_{0}, \omega^{\mathbf{A}}, \omega \in\right.$ $\Omega$ ) consisting of a bounded distributive lattice $\mathbf{A}_{0}=(A, \vee, \wedge, 0,1)$ with completely arbitrary auxiliary operations. Such algebras will be referred to as bounded distributive lattice expansions, or DLEs for short. Our new definition of the canonical extension $\mathbf{A}^{\sigma}$ of a DLE A agrees with the earlier ones whenever they apply.

For a DL $\mathbf{A}=(A, \vee, \wedge, 0,1)$, the canonical extension $\mathbf{A}^{\sigma}=\left(A^{\sigma}, \vee, \wedge, 0,1\right)$ is, as before, a doubly algebraic distributive lattice that contains $\mathbf{A}$ as a (1)

Received October 17, 2001; in revised form July 16, 2002. 
separating and (2) compact sublattice. To say that $\mathbf{A}$ is separating in $\mathbf{A}^{\sigma}$ means that every nonempty interval $[p, u]$ in $\mathbf{A}^{\sigma}$ with $p$ completely join irreducible and $u$ completely meet irreducible contains a member of $A$, and $\mathbf{A}$ is said to be compact in $\mathbf{A}^{\sigma}$ if, for every inequality $\wedge X \leq \bigvee Y$ with $X, Y \subseteq A$, there exist finite sets $F \subseteq X$ and $G \subseteq Y$ with $\bigwedge F \leq \bigvee G$. Together, the properties (1) and (2) characterize $\mathbf{A}^{\sigma}$ up to equivalence as an extension of $\mathbf{A}$.

For a DLE $\mathbf{A}=\left(\mathbf{A}_{0}, \omega^{\mathbf{A}}, \omega \in \Omega\right)$ we also have to extend the additional operations. To this end notice that if $\omega^{\mathbf{A}}$ is an $n$-ary operation - a map from $A^{n}$ into $A$ - then $\omega^{\mathbf{A}^{\sigma}}$ needs to be a map from $A^{\sigma n}$ to $A^{\sigma}$. However, since the latices $\mathbf{A}_{0}^{n \sigma}$ and $\mathbf{A}_{0}^{\sigma n}$ are isomorphic the sets $A^{\sigma n}$ and $A^{n \sigma}$ may harmlessly be identified. Thus we may just consider the problem of extending maps $f: \mathbf{A} \rightarrow \mathbf{B}$ between bounded distributive lattices and then take $\mathbf{A}^{\sigma}$ to be $\left(\mathbf{A}_{0}^{\sigma},\left(\omega^{\mathbf{A}}\right)^{\sigma}, \omega \in \Omega\right)$.

The definition of the canonical extension $f^{\sigma}: \mathbf{A}^{\sigma} \rightarrow \mathbf{B}^{\sigma}$ of a map $f: \mathbf{A} \rightarrow \mathbf{B}$ between bounded distributive lattices that we give in this paper differs fundamentally from the earlier ones, allowing us to consider completely arbitrary maps. We make $f^{\sigma}(x)$ depend only on the values of $f$ at points of $A$ that are "near" $x$. This can be made precise, by introducing a topology on $A^{\sigma}$. Following earlier terminology, elements of $A^{\sigma}$ that are meets and joins of subsets of $A$ are said to be closed and open, respectively. We now define $\sigma$, or $\sigma\left(\mathbf{A}^{\sigma}\right)$, to be the topology having as a basis all intervals $[p, u]$ with $p$ closed and $u$ open. From the compactness property it follows that each of the basic open intervals $[p, u]$ contains a member of $A$, so $A$ is dense in $A^{\sigma}$. We can therefore define $f^{\sigma}(x)$ to be the limit inferior of $f(a)$ for $a \in A$ near $x$; more precisely,

$$
f^{\sigma}(x)=\bigvee\{\bigwedge f(X \cap A): x \in X \in \sigma\} .
$$

To see that $f^{\sigma}$ is in fact an extension of $f$, note that, for $a \in A,\{a\}=[a, a]$ is one of the basic open intervals of $\sigma$, and $a$ is therefore an isolated point of $A^{\sigma}$.

This new definition of the canonical extension $f^{\sigma}$ of a DL map $f$ can be made without any reference to topology. However the topology makes the intuitive idea behind the definition clearer. And, more importantly, the topological approach allows us to develop a more transparent and powerful theory of canonical extensions. For one thing, it becomes possible to characterize the extension of maps abstractly in the topological setting. This characterization is in terms of continuity of the extension. Even though the topology used on the domain is the one described above, the characterization in terms of continuity requires a different topology on the codomain. This asymmetry, it turns out, gives the theory of canonical extensions its particular flavor, and it is also responsible for its complexity. Typically, the preservation of an identity by canonical extensions is proved by showing that in certain situations canonical 
extensions commute with the composition of maps. In the topological approach, this normally involves showing that the composition of the canonical extensions is continuous. This is where the presence of different topologies creates complications, for we cannot simply appeal to the elementary fact that the composition of two continuous maps is continuous. Of course this is not a defect in the present technique, but simply reflects the fact that identities are not always preserved. In fact, the several topologies provide an effective tool for analyzing which properties of the maps imply that the identities are preserved - and this in a very general setting. An unexpected dividend is that, even where the earlier definitions apply, the new approach sometimes yields fresh insights, simpler arguments, and even new results.

Canonical extensions will be defined more carefully in the next section. There we also introduce the topologies, and investigate the relationship between topological and algebraic properties of maps and operations. Sections 3 and 4 are devoted to the fundamental problems of showing that, under some general conditions, the canonical extension of a homomorphism is a homomorphism, and that certain classes of DLEs are closed under canonical extensions. The Epilogue briefly mentions possible generalizations of the results and other related issues.

\section{Topologies on canonical extensions}

\subsection{Canonical extensions of bounded distributive lattices}

The canonical extension of a bounded distributive lattice (DL) is a doubly algebraic distributive lattice $\left(\mathrm{DL}^{+}\right)$in which the original lattice is embedded in a very special manner. We list here some basic facts about this concept, referring the reader to [8] for a more detailed account.

A complete lattice $\mathbf{A}$ is said to be doubly algebraic if both $\mathbf{A}$ and its (algebraic) dual $\mathbf{A}^{\alpha}$ are algebraic. For distributive lattices, this is a very strong property, as the next theorem shows. For a complete lattice $\mathbf{A}$, denote by $J^{\infty}(\mathbf{A})$ the set of all completely join irreducible elements of $\mathbf{A}$, and by $M^{\infty}(\mathbf{A})$ the set of all completely meet irreducible elements of $\mathbf{A}$. We also introduce here some related notation that will be used later. By $J_{\omega}^{\infty}(\mathbf{A})$ and $M_{\omega}^{\infty}(\mathbf{A})$ we denote the sets consisting, respectively, of all finite joins of elements of $J^{\infty}(\mathrm{A})$ and of all finite meets of elements of $M^{\infty}(\mathbf{A})$. Observe that 0 is a member of $J_{\omega}^{\infty}(\mathbf{A})$, but not of $J^{\infty}(\mathbf{A})$, and that 1 is a member of $M_{\omega}^{\infty}(\mathbf{A})$, but not of $M^{\infty}(\mathbf{A})$. For convenience we set $J_{1}^{\infty}(\mathbf{A})=J^{\infty}(\mathbf{A}) \cup\{0\}$ and $M_{1}^{\infty}(\mathbf{A})=M^{\infty}(\mathbf{A}) \cup\{1\}$.

Theorem 2.1. Suppose $\mathbf{A}$ is a complete DL. The following conditions are equivalent:

(i) A is doubly algebraic. 
(ii) $\mathbf{A}$ is algebraic, and every element of $\mathbf{A}$ is a join of completely join irreducible elements.

(iii) $\mathbf{A}$ is completely distributive, and every element of $\mathbf{A}$ is the join of completely join irreducible elements.

(iv) For some poset $P, \mathbf{A}$ is isomorphic to the lattice of all isotone maps from $P$ into the two element chain $C_{2}$.

(v) A is isomorphic to the lattice of all isotone maps from $J^{\infty}(\mathbf{A})$ into $C_{2}$.

Proof. See [8].

Since the notion of a doubly algebraic distributive lattice is selfdual, the duals of (ii) and (iii) also characterize these lattices.

From the fact that a $\mathrm{DL}^{+} \mathbf{A}$ is completely distributive it follows that the completely join irreducible elements of $\mathbf{A}$ are completely join prime, and the completely meet irreducible elements are completely meet prime. This in turn implies that there is a natural isomorphism between the posets $J^{\infty}(\mathbf{A})$ and $M^{\infty}(\mathbf{A})$. For future reference, we fix here the notation for this isomorphism.

Definition 2.2. Given a $\mathrm{DL}^{+} \mathbf{A}$, we define $\kappa(p)=\bigvee(A-\uparrow p)$ for all $p \in J^{\infty}(\mathbf{A})$,

Theorem 2.3. For any $\mathrm{DL}^{+} \mathbf{A}$,

$$
\kappa:\left(J^{\infty}(\mathbf{A}), \leq\right) \simeq\left(M^{\infty}(\mathbf{A}), \leq\right)
$$

and the inverse $\kappa^{-1}$ of $\kappa$ is its dual, i.e., $\kappa^{-1}(u)=\bigwedge(A-\downarrow u)$ for all $u \in$ $M^{\infty}(\mathbf{A})$.

Note that, for $p \in J^{\infty}(\mathbf{A}), \kappa(p)$ is the largest element $x \in A$ with $p \not \leq x$, and for $u \in M^{\infty}(\mathbf{A}), \kappa^{-1}(u)$ is the smallest element $y \in A$ with $y \not \leq u$. Another useful observation about the isomorphism $\kappa$ is that, for $p \in J^{\infty}(\mathbf{A})$, the sets $\uparrow p$ and $\downarrow \kappa(p)$ form a partitioning of $A$, and that every partitioning of $A$ into a principal filter and a principal ideal is of this form. The pairs $\uparrow p$ and $\downarrow \kappa(p)$ are therefore precisely the preimages $h^{-1}(1)$ and $h^{-1}(0)$ under complete homomorphisms $h$ from $\mathbf{A}$ onto the two element lattice.

The canonical extension of a DL was described in [8]. Briefly, two dualities between categories are involved, one, $\mathbf{A} \longmapsto \mathbf{A}_{\delta}$ and $\mathbf{S} \longmapsto \mathbf{S}^{\delta}$, between DLs and Priestley spaces, the other, $\mathbf{A} \longmapsto \mathbf{A}_{*}$ and $\mathbf{P} \longmapsto \mathbf{P}^{*}$ between $\mathrm{DL}^{+} \mathrm{s}$ and posets. The forgetful functor that sends each Priestley space $\mathbf{S}=(S, \tau, \leq)$ into its poset reduct $\mathbf{S}^{\diamond}=(S, \leq)$ connects the two pairs. Any DL $\mathbf{A}$ is isomorphic to its second dual $\left(\mathbf{A}_{\delta}\right)^{\delta}$, which is a sublattice of the $\mathrm{DL}^{+}\left(\mathbf{A}_{\delta}\right)^{\diamond *}$. By definition, $\mathbf{A}^{\sigma}$ is the extension of $\mathbf{A}$, unique up to equivalence, for which there exists a 
commutative diagram

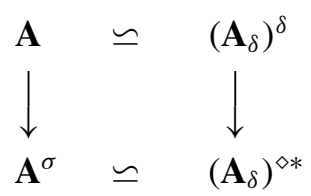

The canonical extension $\mathbf{A}^{\sigma}$ of a DL $\mathbf{A}$ is defined up to equivalence by the condition that the natural isomorphism $\mathbf{A} \simeq\left(\mathbf{A}_{\delta}\right)^{\delta}$ can be extended to an isomorphism $\mathbf{A}^{\sigma} \simeq\left(\mathbf{A}_{\delta}\right)^{\diamond *}$. However, in working with this concept, we will usually make use of two abstract properties that characterize these extensions, rather than going back to details of the construction. It is useful to have names for these properties.

Definition 2.4. Suppose $\mathbf{A}$ is a (bounded) sublattice of a $\mathrm{DL}^{+} \mathbf{A}^{\prime}$. We say that

(i) $\mathbf{A}$ is separating in $\mathbf{A}^{\prime}$ if, for all $p, q \in J^{\infty}\left(\mathbf{A}^{\prime}\right)$ with $p \not \leq q$ there exists $a \in A$ such that $q \leq a$ and $p \not \leq a$.

(ii) $\mathbf{A}$ is compact in $\mathbf{A}^{\prime}$ if, for all $S, T \subseteq A$ with $\bigwedge S \leq \bigvee T$ in $\mathbf{A}^{\prime}$, there exist finite sets $F \subseteq S$ and $G \subseteq T$ such that $\bigwedge F \leq \bigvee G$.

Theorem 2.5. Suppose A is a DL.

(i) $\mathbf{A}^{\sigma}$ is a $\mathrm{DL}^{+}$containing $\mathbf{A}$ as a separating, compact sublattice.

(ii) For any $\mathrm{DL}^{+} \mathbf{A}^{\prime}$ containing $\mathbf{A}$ as a separating, compact sublattice, there exists a unique isomorphism $h: \mathbf{A}^{\sigma} \simeq \mathbf{A}^{\prime}$ sending each member of $A$ into itself.

Proof. See [8].

Again suppose the DL $\mathbf{A}$ is a sublattice of a $\mathrm{DL}^{+} \mathbf{A}^{\prime}$. If the element $x \in A^{\prime}$ is the meet of a subset of $A$, then we say that $x$ is closed and write $x \in$ $K\left(\mathbf{A}^{\prime}\right)$, but if $x$ is the join of a subset of $A$, then we say that $x$ is open and write $x \in O\left(\mathbf{A}^{\prime}\right)$. We say that $x$ is clopen if $x$ is both closed and open. The condition that $\mathbf{A}$ be separating in $\mathbf{A}^{\prime}$ holds if and only if $J^{\infty}\left(\mathbf{A}^{\prime}\right) \subseteq K\left(\mathbf{A}^{\prime}\right)$, or equivalently, $J_{\omega}^{\infty}\left(\mathbf{A}^{\prime}\right) \subseteq K\left(\mathbf{A}^{\prime}\right)$. These conditions are in turn equivalent to their duals, $M^{\infty}\left(\mathbf{A}^{\prime}\right) \subseteq O\left(\mathbf{A}^{\prime}\right)$ and $M_{\omega}^{\infty}\left(\mathbf{A}^{\prime}\right) \subseteq O\left(\mathbf{A}^{\prime}\right)$.

\subsection{Six topologies}

We want to extend a map between DLs (briefly, a DL map) $f: \mathbf{A} \rightarrow \mathbf{B}$ to a map $f^{\sigma}: \mathbf{A}^{\sigma} \rightarrow \mathbf{B}^{\sigma}$ in a way that preserves important properties of the original map. The idea is to define $f^{\sigma}(x)$ as a limit of values of $f$ at elements of $A$ "near" $x$. To make this precise, we need a topology on $A^{\sigma}$. Of the six topologies defined below, the strongest one, $\sigma$, will be used for this purpose, 
but the other five will also be useful in proving preservation properties for canonical extensions.

Definition 2.6. Suppose $\mathbf{A}$ is a DL. We denote by $\sigma, \sigma^{\uparrow}$ and $\sigma^{\downarrow}$ the topologies on $A^{\sigma}$ having as bases, respectively, the sets of the forms $\uparrow p \cap \downarrow u$, $\uparrow p$ and $\downarrow u$, with $p \in K\left(\mathbf{A}^{\sigma}\right)$ and $u \in O\left(\mathbf{A}^{\sigma}\right)$.

When necessary, we write $\sigma\left(\mathbf{A}^{\sigma}\right)$ for $\sigma$, and similarly for the other topologies just defined, as well as for the three topologies defined below. Unlike the three topologies above, the ones defined below are intrinsic to $\mathbf{A}^{\sigma}$; that is, they do not depend on A. In fact, they can be defined on an arbitrary doubly algebraic distributive lattice $\mathbf{B}$, using the set $J_{\omega}^{\infty}(\mathbf{B})$ of finite joins of completely join irreducible elements, and the set $M_{\omega}^{\infty}(\mathbf{B})$ of finite meets of completely meet irreducible elements. Observe that the members of $J_{\omega}^{\infty}(\mathbf{B})$ are the compact elements, in the sense of lattice theory, while the members of $M_{\omega}^{\infty}(\mathbf{B})$ are the dually compact elements.

Definition 2.7. Suppose $\mathbf{B}$ is a doubly algebraic distributive lattice. We denote by $\iota, \iota^{\uparrow}$ and $\iota^{\downarrow}$ the topologies on $B$ having as bases, respectively, the sets of the forms $\uparrow p \cap \downarrow u, \uparrow p$ and $\downarrow u$, with $p \in J_{\omega}^{\infty}(\mathbf{B})$ and $u \in M_{\omega}^{\infty}(\mathbf{B})$.

Obviously, the topology $\sigma$ is the join of $\sigma^{\uparrow}$ and $\sigma^{\downarrow}$, and $\iota$ is the join of $\iota^{\uparrow}$ and $\iota^{\downarrow}$. For any DL $\mathbf{A}$, the topologies $\iota, \iota^{\uparrow}$ and $\iota^{\downarrow}$ on $\mathbf{A}^{\sigma}$ are weaker than $\sigma, \sigma^{\uparrow}$ and $\sigma^{\downarrow}$, respectively, because $J_{\omega}^{\infty}\left(\mathbf{A}^{\sigma}\right) \subseteq K\left(\mathbf{A}^{\sigma}\right)$ and $M_{\omega}^{\infty}\left(\mathbf{A}^{\sigma}\right) \subseteq O\left(\mathbf{A}^{\sigma}\right)$.

It should be noted that $\iota, \iota^{\uparrow}$ and $\iota^{\downarrow}$ are special cases of topologies that have been defined on arbitrary partially ordered sets. In fact, when defined on a $\mathrm{DL}^{+} \mathbf{B}$, both the lower topology and the Scott topology coincide with $\iota^{\uparrow}$. The interval topology (the join of the lower topology and its dual), and the biScott topology (the join of the Scott topology and its dual) therefore both coincide with $\iota$ in this case. For information about these more general topologies see [11]. However, with one exception, no reference will be made to the general theory, for the special cases used here are so much simpler that it is easier to develop the results needed independently.

The connection between the topologies $\sigma$ and $\iota$ and the algebraic structures on the underlying sets is described in part in the next theorem. Part (i) of this theorem is known. In fact, it is shown in [19] that, for any $\mathrm{DL}^{+} \mathbf{B}$, the interval topology is the unique topology that turns $(B, \leq)$ into a Priestley space.

THEOREM 2.8.

(i) For any $\mathrm{DL}^{+} \mathbf{B},(B, \iota, \leq)$ is a Priestley space.

(ii) For any $\mathrm{DL} \mathbf{A},\left(A^{\sigma}, \sigma, \leq\right)$ is totally order disconnected, the set $A$ is dense in $A^{\sigma}$, and the members of $A$ are exactly the isolated points of $A^{\sigma}$. 
Proof. (i) Given $x, y \in B$ with $x \not \leq y$, there exists $p \in J^{\infty}$ (B) with $p \leq x$ and $p \not \leq y$. The filter $\uparrow p$ and the ideal $\downarrow \kappa(p)$ are open sets that form a partitioning of $B$. Since $x$ is in the filter and $y$ is in the ideal, this shows that the space is totally order disconnected. That $(B, \iota)$ is compact, follows from the fact that the Lawson space of any continuous lattice is compact, see [11, Theorem III.1.10].

(ii) By (i), $\left(A^{\sigma}, \iota, \leq\right)$ is totally order disconnected, and since the topology $\sigma$ is stronger than $\iota$, it follows that $\left(A^{\sigma}, \sigma, \leq\right)$ is also totally order disconnected. To show that $A$ is dense in $A^{\sigma}$, it suffices to show that each of the basic intervals $[p, u]$ with $p \leq u, p$ closed and $u$ open, contains a member of $A$. The closed element $p$ and the open element $u$ have representations $p=\bigwedge X$ and $u=\bigvee Y$, with $X, Y \subseteq A$. From the inclusion $\bigwedge X \leq \bigvee Y$ it follows that $\bigwedge F \leq \bigvee G$ for some finite subsets $F$ of $X$ and $G$ of $Y$. Taking $a$ to be either $\bigwedge F$ or $\bigvee G$, we conclude that $a$ is a member of $A$ that belongs to $[p, u]$. Finally, for $a \in A$, the interval $[a, a]=\{a\}$ is open, and $a$ is therefore isolated. On the other hand, since $A$ is dense in $\left(A^{\sigma}, \sigma\right)$, it follows that if $\{x\}$ is open then $x \in A$.

For a DL $\mathbf{A}=(A, \vee, \wedge, 0,1)$, let $\mathbf{A}^{\alpha}$ denote the dual lattice, obtained by reversing the underlying order. That is $\mathbf{A}^{\alpha}=(A, \wedge, \vee, 1,0)$. Since we will often have to change our perspective by replacing a DL by its dual, it is important to realize what effect this has on various derived concepts. Obviously $\mathbf{A}$ and $\mathbf{A}^{\alpha}$ have the same universe. Since the characterization of canonical extensions is selfdual, we may also identify $\mathbf{A}^{\alpha \sigma}$ with $\mathbf{A}^{\sigma \alpha}$, and this leads to several other identifications. We list some important ones. The operation ${ }^{\alpha}$

- leaves the universe unchanged: $A^{\alpha}=A$.

- commutes with $^{\sigma}: \mathbf{A}^{\alpha \sigma}=\mathbf{A}^{\sigma \alpha}$, or equivalently, $\mathbf{A}^{\alpha \sigma \alpha}=\mathbf{A}^{\sigma}$.

- interchanges open elements and closed elements: $K\left(\mathbf{A}^{\alpha \sigma}\right)=O\left(\mathbf{A}^{\sigma}\right)$ and $O\left(\mathbf{A}^{\alpha \sigma}\right)=K\left(\mathbf{A}^{\sigma}\right)$.

- leaves the topologies $\sigma$ and $\iota$ unchanged: $\sigma\left(\mathbf{A}^{\alpha \sigma}\right)=\sigma\left(\mathbf{A}^{\sigma}\right)$ and $\iota\left(\mathbf{A}^{\alpha \sigma}\right)=$ $\iota\left(\mathbf{A}^{\sigma}\right)$.

- interchanges the topologies $\sigma^{\uparrow}$ and $\sigma^{\downarrow}$ as well as $\iota^{\uparrow}$ and $\iota^{\downarrow}: \sigma^{\uparrow}\left(\mathbf{A}^{\alpha \sigma}\right)=$ $\sigma^{\downarrow}\left(\mathbf{A}^{\sigma}\right), \sigma^{\downarrow}\left(\mathbf{A}^{\alpha \sigma}\right)=\sigma^{\uparrow}\left(\mathbf{A}^{\sigma}\right), \iota^{\uparrow}\left(\mathbf{A}^{\alpha \sigma}\right)=\iota^{\downarrow}\left(\mathbf{A}^{\sigma}\right)$, and $\iota^{\downarrow}\left(\mathbf{A}^{\alpha \sigma}\right)=$ $\iota^{\uparrow}\left(\mathbf{A}^{\sigma}\right)$.

Direct products give rise to similar identifications:

- $(\mathbf{A} \times \mathbf{B})^{\sigma}=\mathbf{A}^{\sigma} \times \mathbf{B}^{\sigma}$.

- $K\left((\mathbf{A} \times \mathbf{B})^{\sigma}\right)=K\left(\mathbf{A}^{\sigma}\right) \times K\left(\mathbf{B}^{\sigma}\right)$ and $O(\mathbf{A} \times \mathbf{B})^{\sigma}=O\left(\mathbf{A}^{\sigma}\right) \times O\left(\mathbf{B}^{\sigma}\right)$. 
- $\left.\sigma\left((\mathbf{A} \times \mathbf{B})^{\sigma}\right)=\sigma\left(\mathbf{A}^{\sigma}\right) \times \sigma\left(\mathbf{B}^{\sigma}\right)\right)$, where $\sigma\left(\mathbf{A}^{\sigma}\right) \times \sigma\left(\mathbf{B}^{\sigma}\right)$ is the product topology generated by the two topologies. Similarly for the other five topologies.

As the next theorem shows, it is rather exceptional for the space $\left(A^{\sigma}, \sigma\left(\mathbf{A}^{\sigma}\right)\right)$ to be compact.

Theorem 2.9. For any DL $\mathbf{A}$, the following conditions are equivalent:

(i) The space $\left(A^{\sigma}, \sigma\left(\mathbf{A}^{\sigma}\right)\right)$ is compact.

(ii) $\sigma\left(\mathbf{A}^{\sigma}\right)=\iota\left(\mathbf{A}^{\sigma}\right)$.

(iii) $K\left(\mathbf{A}^{\sigma}\right)=J_{\omega}^{\infty}\left(\mathbf{A}^{\sigma}\right)$ and $O\left(\mathbf{A}^{\sigma}\right)=M_{\omega}^{\infty}\left(\mathbf{A}^{\sigma}\right)$.

Proof. Obviously (ii) implies (i) and (iii) implies (ii).

It is known that, in the poset of all Hausdorff topologies on a set $X$, the compact topologies are minimal (see for example [5, Corollary 3.1.14]). Hence, if $\iota\left(\mathbf{A}^{\sigma}\right)<\sigma\left(\mathbf{A}^{\sigma}\right)$, then $\sigma\left(\mathbf{A}^{\sigma}\right)$ cannot be compact. Thus (i) and (ii) are equivalent.

If (ii) holds, then for all $p \in K\left(\mathbf{A}^{\sigma}\right)$, the filter $\uparrow p$ is the union of basic open sets for the $\iota$-topology. These sets can be taken to be filters $\uparrow q$, with $q$ running through some set $Q \subseteq J_{\omega}^{\infty}\left(\mathbf{A}^{\sigma}\right)$. For each $q \in Q$, we have $\uparrow q \subseteq \uparrow p$, hence $p \leq q$, This inclusion cannot be strict for all $q \in Q$, for then $p$ would not belong to the union of the filters $\uparrow q$. Hence $p \in Q \subseteq J_{\omega}^{\infty}\left(\mathbf{A}^{\sigma}\right)$. Similarly, every member of $O\left(\mathbf{A}^{\sigma}\right)$ belongs to $M_{\omega}^{\infty}\left(\mathbf{A}^{\sigma}\right)$. Thus (ii) implies (iii).

The three conditions are therefore equivalent.

When a topology is presented by specifying a basis $\mathscr{B}$ of open sets, then the isolated points are precisely the points $p$ with $\{p\} \in \mathscr{B}$. In Theorem 2.8 this was used to show that the members of $A$ are exactly the isolated points in the $\sigma$-topology on $A^{\sigma}$. For the $\iota$-topology this shows that the isolated points are the elements that are both joins of finitely many completely join irreducible elements and meets of finitely many completely meet irreducible elements. In general, this will not include all the members of $A$.

\subsection{Continuous extensions of maps}

In defining and investigating extensions of maps $f: \mathbf{A} \rightarrow \mathbf{B}$ between DLs to maps between their canonical extensions, we make use of the various topologies on $A^{\sigma}$ and $B^{\sigma}$. Since several topologies have been defined on each set, it is often necessary to specify which ones are under consideration. In general, if $\tau$ and $\mu$ are topologies on the sets $X$ and $Y$, and if the map $f: X \rightarrow Y$ is continuous relative to $\tau$ on $X$ and $\mu$ on $Y$, then we say that $f$ is $(\tau, \mu)$-continuous, or that $f:(X, \tau) \rightarrow(Y, \mu)$ is continuous. 
Definition 2.10. Suppose $(X, \tau)$ is a topological space, $X^{\prime}$ is a dense subset of $X$, and $\mathbf{C}$ is a $\mathrm{DL}^{+}$. For any map $f: X^{\prime} \rightarrow C$, and for all $x \in X$, we define

$$
\begin{aligned}
& \underline{\lim }_{\tau} f(x)=\bigvee\left\{\bigwedge f\left(U \cap X^{\prime}\right): x \in U \in \tau\right\}, \\
& \overline{\lim }_{\tau} f(x)=\bigwedge\left\{\bigvee f\left(U \cap X^{\prime}\right): x \in U \in \tau\right\} .
\end{aligned}
$$

When the intended topology is clear from the context, the subscript $\tau$ will usually be omitted.

In the above definition, $U$ runs through all open neighborhoods of $x$, but we can instead take the values of $U$ to be the members of some fixed neighborhood basis. This observation will be used frequently below.

TheOREM 2.11. Let $(X, \tau), X^{\prime}, \mathbf{C}$ and $f$ be as in the preceding definition. The following statements hold.

(i) $\underline{\lim } f:(X, \tau) \rightarrow\left(C, \iota^{\uparrow}\right)$ is continuous.

(ii) $\varlimsup_{\lim } f:(X, \tau) \rightarrow\left(C, \iota^{\downarrow}\right)$ is continuous.

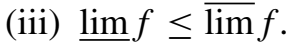

(iv) $\lim f \uparrow X^{\prime} \leq f \leq \varlimsup \lim f \uparrow X^{\prime}$.

(v) For all $x \in X^{\prime}, \underline{\lim } f(x)=f(x)$ if and only if $f:\left(X^{\prime}, \tau \uparrow X^{\prime}\right) \rightarrow\left(C, \iota^{\uparrow}\right)$ is continuous at $x$.

(vi) For all $x \in X^{\prime}, \overline{\lim } f(x)=f(x)$ ifand only if $f:\left(X^{\prime}, \tau \uparrow X^{\prime}\right) \rightarrow\left(C, \iota^{\downarrow}\right)$ is continuous at $x$.

(vii) Suppose $g: X \rightarrow C$ is $\left(\tau, \iota^{\uparrow}\right)$-continuous at $x$ and $g(x) \leq f(x)$.

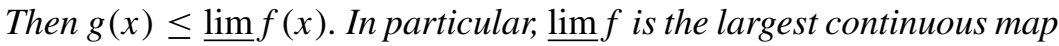
$g:(X, \tau) \rightarrow\left(C, \iota^{\uparrow}\right)$ with $g \uparrow X^{\prime} \leq f$.

(viii) Suppose $g: X \rightarrow C$ is $\left(\tau, \iota^{\downarrow}\right)$-continuous at $x$ and $g(x) \geq f(x)$. Then $g(x) \geq \overline{\lim } f(x)$. In particular, $\varlimsup$ 两 $f$ is the smallest continuous map $g:(X, \tau) \rightarrow\left(C, \iota^{\downarrow}\right)$ with $g \uparrow X^{\prime} \geq f$.

(ix) For all $x \in X, \underline{\lim } f(x)=\varlimsup \operatorname{\operatorname {lim}} f(x)$ if and only if $\underline{\lim f}$ is $\left(\tau, \iota^{\downarrow}\right)$ continuous at $x$, if and only if $\overline{\lim } f$ is $\left(\tau, \iota^{\uparrow}\right)$-continuous at $x$.

Proof. To prove (i) it suffices to show that, for every $p \in J^{\infty}(\mathbf{C})$, the inverse image of the filter $\uparrow p$ under $\lim f$ is open. Now, for any $x \in X$, if $\underline{\lim } f(x) \in \uparrow p$, then

$$
p \leq \bigvee\left\{\bigwedge f\left(U \cap X^{\prime}\right): x \in U \in \tau\right\}
$$

Since $p \in J^{\infty}(\mathbf{C})$, there is $U_{0} \in \tau$ with $x \in U_{0}$ and $p \leq \bigwedge f\left(U_{0} \cap X^{\prime}\right)$, but from this it readily follows that $\underline{\lim f}$ sends the whole neighborhood $U_{0}$ of $x$ 
into $\uparrow p$. Statement (ii) follows by duality, and (iii) and (iv) are clear from the definitions of $\underline{\lim f}$ and $\varlimsup \lim f$.

To say that $f:\left(X^{\prime}, \tau\left\lceil X^{\prime}\right) \rightarrow\left(C, \iota^{\uparrow}\right)\right.$ is continuous at $x$ means that, for every $p \in J^{\infty}(\mathbf{C})$ below $f(x), f$ sends some $\tau\left\lceil X^{\prime}\right.$-neighborhood of $x$ into $\uparrow p$, but this holds if and only if $\underline{\lim } f(x) \geq f(x)$. Since the inequality $\underline{\lim } f(x) \leq f(x)$ holds for all $x \in X^{\prime}$, this proves (v), and (vi) follows by duality.

To prove (vii), we consider a function $g: X \rightarrow C$ and $x \in X$ with $g(x) \leq f(x)$ and $g\left(\tau, \iota^{\uparrow}\right)$-continuous at $x$, and show that $g(x) \leq \underline{\lim } f(x)$. Given $p \in J^{\infty}(\mathbf{C})$ with $p \leq g(x)$, it follows from the continuity of $g$ that $g$ takes some neighborhood $U$ of $x$ into $\uparrow p$. Hence $f$ takes $U \cap X^{\prime}$ into $\uparrow p$, which implies that $p \leq \underline{\lim } f(x)$. This proves (vii), and (viii) follows by duality.

Statement (ix) now readily follows. In general, $\underline{\lim f \leq \varlimsup \lim f} f$ If $\underline{\lim f}$ is $\left(\tau, \iota^{\downarrow}\right)$-continuous at $x$, then we apply (vii) with $g=\underline{\lim f}$ to infer that the two limits agree at $x$. The converse holds because $\overline{\lim } f$ is $\left(\tau, \iota^{\downarrow}\right)$-continuous. The argument with the two limits interchanged is similar.

Theorem 2.12. Suppose $\mathbf{A}$ is a $\mathrm{DL}$ and $\mathbf{C}$ is a $\mathrm{DL}^{+}$. For any map $f: A \rightarrow$ $C$, the maps $\underline{\lim }_{\sigma} f, \varlimsup_{\lim _{\sigma}} f: A^{\sigma} \rightarrow C$ are, respectively, the largest $\left(\sigma, \iota^{\uparrow}\right)$ continuous extension of $f$ and the smallest $\left(\sigma, \iota^{\downarrow}\right)$-continuous extension of $f$.

Proof. Recall that every element $a$ of $A$ is an isolated point of $A^{\sigma}$, so that $\{a\} \in \sigma\left(\mathbf{A}^{\sigma}\right)$. Hence $\underline{\lim f}$ and $\varlimsup \lim f$ restricted to $A$ are both $(\sigma, \tau)$-continuous for any $\tau$, and $\underline{\lim } f(a)$ and $\overline{\lim } f(a)$ are both equal to $f(a)$. Thus $\underline{\lim } f$ and $\varlimsup \lim f$ are both extensions of $f$. The theorem now follows readily from parts (vii) and (viii) of the preceding theorem.

\subsection{Canonical extensions as continuous extensions}

We now define canonical extensions of arbitrary maps between DLs.

Definition 2.13. For any DL map $f: \mathbf{A} \rightarrow \mathbf{B}$, we define

$$
f^{\sigma}=\underline{\lim }_{\sigma} f, \quad f^{\pi}=\varlimsup_{\overline{\lim _{\sigma}}} f .
$$

Theorem 2.14. For any DL map $f: \mathbf{A} \rightarrow \mathbf{B}$, and for all $x \in A^{\sigma}$,

$$
\begin{aligned}
& f^{\sigma}(x)=\bigvee\left\{\bigwedge f([p, u] \cap A): p \in K\left(\mathbf{A}^{\sigma}\right), u \in O\left(\mathbf{A}^{\sigma}\right), \text { and } p \leq x \leq u\right\} \\
& f^{\pi}(x)=\bigwedge\left\{\bigvee f([p, u] \cap A): p \in K\left(\mathbf{A}^{\sigma}\right), u \in O\left(\mathbf{A}^{\sigma}\right), \text { and } p \leq x \leq u\right\}
\end{aligned}
$$

The following special case of Theorem 2.12 will play an important role. 
Theorem 2.15. For any DL map $f: \mathbf{A} \rightarrow \mathbf{B}, f^{\sigma}$ and $f^{\pi}$ are extensions of $f$. In fact, $f^{\sigma}$ is the largest $\left(\sigma, \iota^{\uparrow}\right)$-continuous extension of $f$ to $A^{\sigma}$, and $f^{\pi}$ is the smallest $\left(\sigma, \iota^{\downarrow}\right)$-continuous extension of $f$ to $A^{\sigma}$.

As may be expected, the theory behaves well for maps whose two canonical extensions coincide. We give a name to such maps.

Definition 2.16. A DL map $f: \mathbf{A} \rightarrow \mathbf{B}$ is said to be smooth if $f^{\sigma}=f^{\pi}$.

Corollary 2.17. If the DL map $f: \mathbf{A} \rightarrow \mathbf{B}$ is smooth, then the extension $f^{\sigma}=f^{\pi}$ of $f$ is $(\sigma, \iota)$-continuous. Conversely, if $f$ has a $(\sigma, \iota)$-continuous extension $g: \mathbf{A}^{\sigma} \rightarrow \mathbf{B}^{\sigma}$, then $f$ is smooth and $f^{\sigma}=f^{\pi}=g$.

Proof. If $f^{\sigma}$ and $f^{\pi}$ are equal to the same map $g$, then by Theorem 2.15, $g$ is both the largest $\left(\sigma, \iota^{\uparrow}\right)$-continuous extension of $f$ and the smallest $\left(\sigma, \iota^{\downarrow}\right)$ continuous extension of $f$. It follows that $g$ is $(\sigma, \iota)$-continuous, and is the only extension of $f$ with that property.

Conversely, suppose $g: \mathbf{A}^{\sigma} \rightarrow \mathbf{B}^{\sigma}$ is a $(\sigma, \iota)$-continuous extension of $f$. Then, by Theorem 2.15, $g \leq f^{\sigma}$ and $f^{\pi} \leq g$, so all three maps are equal.

For isotone maps, the new definition of canonical extension agrees with the earlier one.

Corollary 2.18. If the $\mathrm{DL}$ map $f: \mathbf{A} \rightarrow \mathbf{B}$ is isotone, then for all $x \in A^{\sigma}$,

$$
\begin{aligned}
& f^{\sigma}(x)=\bigvee\left\{\bigwedge f(\uparrow p \cap A): x \geq p \in K\left(\mathbf{A}^{\sigma}\right)\right\}, \\
& f^{\pi}(x)=\bigwedge\left\{\bigvee f(\downarrow u \cap A): x \leq u \in O\left(\mathbf{A}^{\sigma}\right)\right\} .
\end{aligned}
$$

Proof. This follows from the fact that in this case $\bigwedge f([p, u] \cap A)=$ $\bigwedge f(\uparrow p \cap A)$ and $\bigvee f([p, u] \cap A)=\bigvee f(\downarrow u \cap A)$ for $p \in K\left(\mathbf{A}^{\sigma}\right)$ and $u \in O\left(\mathbf{A}^{\sigma}\right)$ with $p \leq u$.

Theorem 2.19. If the DL map $f: \mathbf{A} \rightarrow \mathbf{B}$ is isotone, then

(i) $f^{\sigma}(p)=\bigwedge\{f(a): a \in \uparrow p \cap A\}$ for all $p \in K\left(\mathbf{A}^{\sigma}\right)$,

(ii) $f^{\pi}(u)=\bigvee\{f(a) ; a \in \downarrow u \cap A\}$ for all $u \in O\left(\mathbf{A}^{\sigma}\right)$,

(iii) $f^{\sigma}(x)=\bigvee\left\{f^{\sigma}(p): p \in \downarrow x \cap K\left(\mathbf{A}^{\sigma}\right)\right\}$ for all $x \in A^{\sigma}$,

(iv) $f^{\pi}(x)=\bigwedge\left\{f^{\pi}(u): u \in \uparrow x \cap O\left(\mathbf{A}^{\sigma}\right)\right\}$ for all $x \in A^{\sigma}$.

Proof. The first two formulas follow from the preceding corollary and the fact that $\uparrow p$ is the smallest filter containing $p$ and $\downarrow u$ is the smallest ideal 
containing $u$. Formulas three and four follow from the preceding corollary and the first two formulas.

Theorem 2.20. For any isotone DL map $f: \mathbf{A} \rightarrow \mathbf{B}$,

(i) $f^{\sigma}(x)=f^{\pi}(x)$ for all $x \in K\left(\mathbf{A}^{\sigma}\right) \cup O\left(\mathbf{A}^{\sigma}\right)$.

(ii) $f^{\sigma}$ and $f^{\pi}$ take closed elements into closed elements and open elements into open elements.

(iii) $f^{\sigma}$ and $f^{\pi}$ are $(\sigma, \iota)$-continuous at every point of $K\left(\mathbf{A}^{\sigma}\right) \cup O\left(\mathbf{A}^{\sigma}\right)$.

Proof. If $p \in K\left(\mathbf{A}^{\sigma}\right)$, then $f^{\pi}(p)$ is the meet of all $f^{\pi}(u)$ with $p \leq u \in$ $O\left(\mathbf{A}^{\sigma}\right)$. For each such element $u$, there exists $a \in A$ with $p \leq a \leq u$, and hence $f^{\pi}(p) \leq f(a) \leq f^{\pi}(u)$. Therefore, $f^{\pi}(p)=\bigwedge f(\uparrow p \cap A)=f^{\sigma}(p)$. Dually, $f^{\sigma}$ and $f^{\pi}$ agree on the open elements. Thus (i) holds.

By the preceding theorem, $f^{\sigma}$ sends closed elements into closed elements, and $f^{\pi}$ sends open elements into open elements. Since $f^{\sigma}$ and $f^{\pi}$ agree on the closed elements and on the open elements, (ii) follows.

Finally, consider an element $x \in K\left(\mathbf{A}^{\sigma}\right) \cup O\left(\mathbf{A}^{\sigma}\right)$. Then $f^{\sigma}(x)=f^{\pi}(x)$ and, by Theorem 2.12, $f^{\sigma}$ is $\left(\sigma, \iota^{\uparrow}\right)$-continuous at $x$. For any $u \in M^{\infty}\left(\mathbf{A}^{\sigma}\right)$ with $f^{\sigma}(x)=f^{\pi}(x) \leq u$ there is a $\sigma$-neighborhood $U$ of $x$ such that $f^{\pi}(U) \subseteq \downarrow u$. Since $f^{\sigma} \leq f^{\pi}$ it follows that $f^{\sigma}(U) \subseteq \downarrow u$ and thus $f^{\sigma}$ is $\left(\sigma, \iota^{\downarrow}\right)$-continuous at $x$. We have shown that $f^{\sigma}$ is $(\sigma, \iota)$-continuous at $x$. By duality $f^{\pi}$ is $(\sigma, \iota)$ continuous at $x$ as well.

A DL map $f: \mathbf{A} \rightarrow \mathbf{B}$ is said to be join preserving, or to preserve joins, if it preserves all binary joins, and hence all nonempty finite joins. Assuming that $\mathbf{A}$ and $\mathbf{B}$ are complete, $f$ is said to be completely join preserving if it preserves all nonempty joins. Observe that it is not required that $f$ preserve the empty join, i.e., that $f(0)=0$. The corresponding notions for meets are defined dually. A map that is (completely) join and meet preserving is called a (complete) lattice homomorphism, while a (complete) DL homomorphism is also required to take 0 to 0 and 1 to 1 .

We will be considering canonical extensions of $n$-ary operations on a DL A, i.e., maps $f: \mathbf{A}^{n} \rightarrow \mathbf{A}$. It is convenient to consider, more generally, maps

$$
f: \mathbf{A}_{0} \times \mathbf{A}_{1} \times \cdots \times \mathbf{A}_{n-1} \rightarrow \mathbf{B}
$$

where $\mathbf{A}_{0}, \mathbf{A}_{1}, \ldots, \mathbf{A}_{n-1}$ and $\mathbf{B}$ are DLs. With the obvious identification

$$
\left(\mathbf{A}_{0} \times \mathbf{A}_{1} \times \cdots \times \mathbf{A}_{n-1}\right)^{\sigma}=\mathbf{A}_{0}^{\sigma} \times \mathbf{A}_{1}^{\sigma} \times \cdots \times \mathbf{A}_{n-1}^{\sigma},
$$

the canonical extension of $f$ may be viewed as a map

$$
f^{\sigma}: \mathbf{A}_{0}^{\sigma} \times \mathbf{A}_{1}^{\sigma} \times \cdots \times \mathbf{A}_{n-1}^{\sigma} \rightarrow \mathbf{B}^{\sigma} .
$$


In particular, the canonical extension of an $n$-ary operation on a DL $\mathbf{A}$ becomes an $n$-ary operation on $\mathbf{A}^{\sigma}$.

In [15], an operation on a Boolean algebra was called an operator if it preserves binary joins in each of its coordinates, and in [8] this terminology was extended to operations on DLs. We now extend this terminology to maps of the form (2.1). As before, the operator is said to be complete if the DLs are complete and the operator preserves all nonempty joins in each of its coordinates. The basic result that the canonical extension of an operator is a complete operator, Theorem 2.4 in [15], carries over to this more general setting.

THEOREM 2.21. The canonical extension of an operator is a complete operator. In particular, the canonical extension of a join preserving map is completely join preserving.

The proof in [15] applies with no significant modifications. However, we give here a shorter proof that yields a somewhat more general result.

LEMMA 2.22. For any isotone DL map

$$
f: \mathbf{A}_{0} \times \mathbf{A}_{1} \rightarrow \mathbf{B}
$$

if $f$ preserves joins in the first coordinate, then

$$
f^{\sigma}: \mathbf{A}_{0}^{\sigma} \times \mathbf{A}_{1}^{\sigma} \rightarrow \mathbf{B}^{\sigma}
$$

preserves arbitrary nonempty joins in the first coordinate.

Proof. It suffices to show that, for any $x_{0} \in A_{0}^{\sigma}$ and $x_{1} \in A_{1}^{\sigma}$, and for any $p \in J^{\infty}\left(\mathbf{B}^{\sigma}\right)$,

$$
p \leq f^{\sigma}\left(x_{0}, x_{1}\right) \text { implies }(\exists q \in Q) p \leq f^{\sigma}\left(q, x_{1}\right),
$$

where $Q=\left\{q \in J_{1}^{\infty}\left(\mathbf{A}_{0}^{\sigma}\right): q \leq x_{0}\right\}$. From the fact that $f$ is isotone it follows that $f^{\sigma}\left(x_{0}, x_{1}\right)$ is the join of all the elements $f^{\sigma}\left(y_{0}, y_{1}\right)$ with $x_{i} \geq y_{i} \in K\left(\mathbf{A}_{i}\right)$ for $i=0,1$. We may therefore assume that $x_{0}$ and $x_{1}$ are closed.

Assume now that (2.2) fails. Then there exist, for each $q \in Q$, elements $a_{q, 0} \geq q$ and $a_{q, 1} \geq x_{1}$ in $A$ such that $p \not \leq f\left(a_{q, 0}, a_{q, 1}\right)$. We have $x_{0}=$ $\bigvee Q \leq \bigvee\left\{a_{q, 0}: q \in Q\right\}$, and hence $x_{0} \leq \bigvee\left\{a_{q, 0}: q \in F\right\}$ for some finite $F \subseteq Q$. The join $a_{0}$ of the elements $a_{q, 0}$ with $q \in F$, and the meet $a_{1}$ of the corresponding elements $a_{q, 1}$ are in $A$. Clearly $p \not \leq f\left(a_{q, 0}, a_{1}\right)$ for each $q \in F$. Since $f$ preserves joins in its first coordinate, it follows that $p \not \leq f\left(a_{0}, a_{1}\right)$ and hence $p \not \leq f^{\sigma}\left(x_{0}, x_{1}\right)$.

A map $f: \mathbf{C} \rightarrow \mathbf{D}$ between complete lattices is said to be upper continuous if $f(\bigvee S)=\bigvee f(S)$ for every updirected set $S \subseteq C$. If $\mathbf{C}$ and $\mathbf{D}$ are $\mathrm{DL}^{+} \mathrm{s}$, 
then this holds if and only if, for all $x \in C$ and $p \in J^{\infty}(\mathbf{D})$ with $f(x) \geq p$, there exists $q \in J_{\omega}^{\infty}(\mathbf{C})$ such that $q \leq x$ and $f(q) \geq p$. In other words, a DL ${ }^{+}$ map is upper continuous if and only if it is $\left(\iota^{\uparrow}, \iota^{\uparrow}\right)$-continuous.

Theorem 2.23. If $\mathbf{C}_{0}, \mathbf{C}_{1}, \ldots, \mathbf{C}_{n-1}$ and $\mathbf{D}$ are $\mathrm{DL}^{+} s$, then every complete operator $f: \mathbf{C}_{0} \times \mathbf{C}_{1} \times \cdots \times \mathbf{C}_{n-1} \rightarrow \mathbf{D}$ is upper continuous.

Proof. Given $x=\left(x_{0}, x_{1}, \ldots, x_{n-1}\right) \in \mathbf{C}_{0} \times \mathbf{C}_{1} \times \cdots \times \mathbf{C}_{n-1}$ and $p \in$ $J^{\infty}(\mathbf{D})$ with $f(x) \geq p$, we can always pick $q=\left(q_{0}, q_{1}, \ldots, q_{n-1}\right)$ with $x_{i} \geq q_{i} \in J_{1}^{\infty}\left(\mathbf{C}_{i}\right)$ for $i<n$ and $f(q) \geq p$.

The canonical extension $f^{\sigma}$ of an arbitrary DL map $f: \mathbf{A} \rightarrow \mathbf{B}$ has been shown to be $\left(\sigma, \iota^{\uparrow}\right)$-continuous. When more is known about $f$, more can often be said about the continuity of $f^{\sigma}$. The facts listed in the next theorem will be used extensively. Many other such results suggest themselves; we only list those that will be needed below.

Theorem 2.24. For every DL map $f: \mathbf{A} \rightarrow \mathbf{B}, f^{\sigma}$ is $\left(\sigma, \iota^{\uparrow}\right)$-continuous. Furthermore,

(i) If $f$ is isotone, then $f^{\sigma}$ is $\left(\sigma^{\uparrow}, \iota^{\uparrow}\right)$-continuous;

(ii) If $f$ is an operator, then $f^{\sigma}$ is $\left(\iota^{\uparrow}, \iota^{\uparrow}\right)$-continuous;

(iii) If $f$ is join preserving, then $f^{\sigma}$ is $\left(\sigma^{\downarrow}, \sigma^{\downarrow}\right)$-continuous;

(iv) If $f$ is join and meet preserving, then $f^{\sigma}$ is $(\sigma, \sigma)$-continuous.

Proof. The first conclusion holds by Theorem 2.15 . To prove (i), we simply note that every isotone map $g: \mathbf{A}^{\sigma} \rightarrow \mathbf{B}^{\sigma}$ that is $\left(\sigma, \iota^{\uparrow}\right)$-continuous is $\left(\sigma^{\uparrow}, \iota^{\uparrow}\right)$ continuous. This is so because the counterimage under $g$ of any basic open set $\uparrow p$ is an order filter, and since it is $\sigma$-open, it must be $\sigma^{\uparrow}$-open. If $f$ is an operator, then $f^{\sigma}$ is upper continuous, which is true just in case it is $\left(\iota^{\uparrow}, \iota^{\uparrow}\right)$-continuous.

For (iii), suppose $f$ is join preserving. Then by Theorem $2.22, f^{\sigma}$ is completely join preserving. Hence the counterimage of a principal ideal $\downarrow v$ in $\mathbf{B}^{\sigma}$ is either empty, or else it is a principal ideal $\downarrow u$ in $\mathbf{A}^{\sigma}$. It suffices to show that if $v$ is open, then so is $u$. To prove this, consider a closed element $p \leq u$. Then $\bigwedge f(\uparrow p \cap A)=f^{\sigma}(p) \leq v$. Hence $\bigwedge f(F) \leq v$ for some finite $F \subseteq \downarrow p \cap A$. Letting $a_{p}=\bigwedge F$, we infer that $a_{p}$ is a member of $A$, and that $p \leq a_{p} \leq u$. The element $u$ is therefore the join of all the elements $a_{p}$ with $p$ closed and $p \leq u$, and is therefore open.

Suppose $f$ is join and meet preserving. By (iii), $f^{\sigma}$ is $\left(\sigma^{\downarrow}, \sigma^{\downarrow}\right)$-continuous. Of course, $f^{\sigma}$ is also $\left(\sigma, \iota^{\uparrow}\right)$-continuous, so it is $(\sigma, \iota)$-continuous, which in turn entails that $f$ is smooth. Also, from the fact that $f$ is meet preserving and smooth it follows by the dual of (iii) that $f^{\sigma}=f^{\pi}$ is $\left(\sigma^{\uparrow}, \sigma^{\uparrow}\right)$-continuous. Hence $f^{\sigma}$ is $(\sigma, \sigma)$-continuous. 
Corollary 2.25. If the $\mathrm{DL}$ map $f: \mathbf{A} \rightarrow \mathbf{B}$ is either join preserving or meet preserving, then $f$ is smooth.

Proof. Suppose $f$ is join preserving. By Theorem 2.24(iii), $f^{\sigma}$ is $\left(\sigma^{\downarrow}, \sigma^{\downarrow}\right)$ continuous and hence also $\left(\sigma, \iota^{\downarrow}\right)$-continuous. Like all canonical extensions, $f^{\sigma}$ is $\left(\sigma, \iota^{\uparrow}\right)$-continuous. Hence $f^{\sigma}$ is $(\sigma, \iota)$-continuous. I.e., $f$ is smooth. The case when $f$ is meet preserving follows by duality.

We conclude this subsection with two examples.

EXAMPLE 2.26. A join preserving dual operator need not have a $(\sigma, \sigma)$ continuous canonical extension. Our counterexample is the join operator itself. In any DL $\mathbf{A}, V^{\mathbf{A}}$ is a join preserving dual operator (although it is not meet preserving) and it is easy to see that $\left(\vee^{\mathbf{A}}\right)^{\sigma}=\vee^{\mathbf{A}^{\sigma}}$. Now let $x, y \in A^{\sigma}$ be complementary nonmembers of $A$; such elements will exist, e.g., if $\mathbf{A}$ is an infinite Boolean algebra. Then $x \vee y=1$, but since $x$ and $y$ cannot both be closed, there are no closed elements $p \leq x$ and $q \leq y$ with $p \vee q=1$. Hence the preimage of the $\sigma$-open set $\{1\}$ is not $\sigma$-open.

EXAMPLE 2.27. The canonical extension of a join preserving map need not be $(\iota, \iota)$-continuous.

Let $\mathbf{A}$ be any infinite Boolean algebra, and let $f(0)=0$, and $f(x)=1$ whenever $0 \neq x \in A$. Then $f^{\sigma}(0)=0$, and $f^{\sigma}(x)=1$ whenever $0 \neq x \in$ $A^{\sigma}$. Obviously, $f$ is join preserving, but $f^{\sigma}$ is not $(\iota, \iota)$-continuous, for if $u$ is any coatom of $\mathbf{A}^{\sigma}$, then $\downarrow u$ is an $\iota$-open set whose preimage $\{0\}$ is not $\iota$-open.

\subsection{Composition and canonical extensions}

In many cases, properties can be shown to be preserved by canonical extensions by showing that the commutativity of certain diagrams is preserved. The following question is therefore important: Consider the diagrams
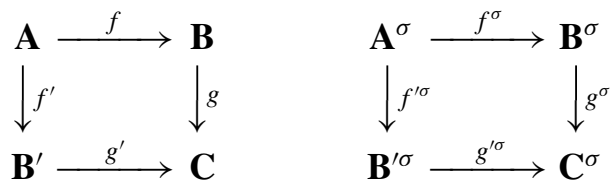

Given that the first diagram commutes, under what conditions does it follow that the second diagram also commutes? The property $(\mathrm{PH})$ of preservation of homomorphisms can be formulated in this manner. Similarly, the preservation of identities reduces to problems of this nature.

An obvious way to try to prove that commutativity of diagrams is preserved is to show that

$$
g^{\sigma} f^{\sigma}=(g f)^{\sigma}=\left(g^{\prime} f^{\prime}\right)^{\sigma}=f^{\prime \sigma} g^{\prime \sigma} .
$$


Here the second equality holds by hypothesis. The question is therefore, when canonical extensions distribute over composition, i.e., under what conditions it is the case that $(g f)^{\sigma}=g^{\sigma} f^{\sigma}$.

As we know from [15] and [8], the inequality $(g f)^{\sigma} \leq g^{\sigma} f^{\sigma}$ holds for an important class of maps.

Theorem 2.28 (Ribeiro [21]). For any DL maps $f: \mathbf{A} \rightarrow \mathbf{B}$ and $g: \mathbf{B} \rightarrow$ $\mathrm{C}$, if $f$ and $g$ are isotone then $(g f)^{\sigma} \leq g^{\sigma} f^{\sigma}$.

Proof. Looking at the formulas

$$
\begin{aligned}
& (g f)^{\sigma}(x)=\bigvee\left\{\bigwedge g f(\uparrow p \cap A): x \geq p \in K\left(\mathbf{A}^{\sigma}\right)\right\}, \\
& g^{\sigma} f^{\sigma}(x)=\bigvee\left\{\bigwedge g(\uparrow q \cap B): f^{\sigma}(x) \geq q \in K\left(\mathbf{B}^{\sigma}\right)\right\},
\end{aligned}
$$

all we need to do is to observe that each of the joinands $\bigwedge g f(\uparrow p \cap A)$ in the first formula is below the joinand $\bigwedge g\left(\uparrow f^{\sigma}(p) \cap B\right)$ in the second. It therefore suffices to show that, for every element $b \in \uparrow f^{\sigma}(p) \cap B$, we can find $a \in \uparrow p \cap A$ with $f(a) \leq b$. Now $f^{\sigma}(p)=\bigwedge f(\uparrow p \cap A)$, so the condition $b \in \uparrow f^{\sigma}(p) \cap B$ implies that, for some finite set $F \subseteq \uparrow p \cap A, \bigwedge f(F) \leq b$, and $\bigwedge F$ is our required element $a$.

Corollary 2.29. For any isotone DL maps $f: \mathbf{A} \rightarrow \mathbf{B}$ and $g: \mathbf{B} \rightarrow \mathbf{C}$,

$$
(g f)^{\sigma} \leq g^{\sigma} f^{\sigma} \leq\left\{\begin{array}{l}
g^{\sigma} f^{\pi} \\
g^{\pi} f^{\sigma}
\end{array}\right\} \leq g^{\pi} f^{\pi} \leq(g f)^{\pi}
$$

with equality holding on $K\left(\mathbf{A}^{\sigma}\right) \cup O\left(\mathbf{A}^{\sigma}\right)$.

Proof. The first inclusion holds by the preceding theorem, and the last one follows by duality. The remaining inclusions follow from the fact that, by Theorem 2.11(iii), $f^{\sigma} \leq f^{\pi}$ for an arbitrary DL map $f$, and the obvious observation that the canonical extension of an isotone map is isotone. Applying Theorem 2.20 to the map $g f$, we see that $(g f)^{\sigma}$ and $(g f)^{\pi}$ agree on $K\left(\mathbf{A}^{\sigma}\right) \cup$ $O\left(\mathbf{A}^{\sigma}\right)$, whence all six functions agree there.

Theorem 2.30. For any DL maps $f: \mathbf{A} \rightarrow \mathbf{B}$ and $g: \mathbf{B} \rightarrow \mathbf{C}$ :

(i) The inequality $g^{\sigma} f^{\sigma} \leq(g f)^{\sigma}$ holds whenever $g^{\sigma} f^{\sigma}$ is $\left(\sigma, \iota^{\uparrow}\right)$-continuous.

(ii) The inequality $g^{\sigma} f^{\sigma} \geq(g f)^{\sigma}$ holds whenever $g^{\sigma} f^{\sigma}$ is $\left(\sigma, \iota^{\downarrow}\right)$-continuous.

(iii) The equation $g^{\sigma} f^{\sigma}=(g f)^{\sigma}$ holds whenever $g^{\sigma} f^{\sigma}$ is $(\sigma, \iota)$-continuous.

Proof. By Theorem 2.15, $(g f)^{\sigma}$ is the largest $\left(\sigma, \iota^{\uparrow}\right)$-continuous extension of $g f$. Hence, if the extension $g^{\sigma} f^{\sigma}$ of $g f$ is also $\left(\sigma, \iota^{\uparrow}\right)$-continuous, then $g^{\sigma} f^{\sigma} \leq(g f)^{\sigma}$. This proves (i). For the proof of (ii) we use the fact that $(g f)^{\pi}$ 
is the smallest $\left(\sigma, \iota^{\downarrow}\right)$-continuous extension of $g f$. Therefore, if $g^{\sigma} f^{\sigma}$ is also $\left(\sigma, \iota^{\downarrow}\right)$-continuous, then $(g f)^{\sigma} \geq(g f)^{\pi} \geq g^{\sigma} f^{\sigma}$. Statement (iii) is just the conjunction of (i) and (ii).

We now make use of the fact that the composition of continuous maps is continuous.

Corollary 2.31. For any DL maps $f: \mathbf{A} \rightarrow \mathbf{B}$ and $g: \mathbf{B} \rightarrow \mathbf{C}:$

(i) If, for some topology $\tau$ on $B^{\sigma}, f^{\sigma}$ is $(\sigma, \tau)$-continuous and $g$ is $\left(\tau, \iota^{\uparrow}\right)$ continuous, then $g^{\sigma} f^{\sigma} \leq(g f)^{\sigma}$. In particular, this holds when $f^{\sigma}$ is $(\sigma, \sigma)$-continuous, and when $g^{\sigma}$ is $\left(\iota^{\uparrow}, \iota^{\uparrow}\right)$-continuous.

(ii) If, for some topology $\tau$ on $B^{\sigma}, f^{\sigma}$ is $(\sigma, \tau)$-continuous and $g^{\sigma}$ is $\left(\tau, \iota^{\downarrow}\right)$ continuous, then $(g f)^{\sigma} \leq g^{\sigma} f^{\sigma}$. In particular, this holds when $f^{\sigma}$ is $(\sigma, \sigma)$-continuous and $g$ is smooth, when $f$ is smooth and $g^{\sigma}$ is $\left(\iota, \iota^{\downarrow}\right)$ continuous, and when $g^{\sigma}$ is $\left(\iota^{\uparrow}, \iota^{\downarrow}\right)$-continuous.

(iii) If, for some topology $\tau$ on $B^{\sigma}, f^{\sigma}$ is $(\sigma, \tau)$-continuous and $g$ is $(\tau, \iota)$ continuous, then $(g f)^{\sigma}=g^{\sigma} f^{\sigma}$. In particular, this holds whenever $f^{\sigma}$ is $(\sigma, \sigma)$-continuous and $g$ is smooth, and when $f$ is smooth and $g^{\sigma}$ is $(\iota, \iota)$-continuous.

Corollary 2.32. For any DL maps $f: \mathbf{A} \rightarrow \mathbf{B}$ and $g: \mathbf{B} \rightarrow \mathbf{C}$, the equality $(g f)^{\sigma}=g^{\sigma} f^{\sigma}$ holds if $f$ and $g$ are isotone and either $f^{\sigma}$ is $(\sigma, \sigma)$ continuous or $g$ is $\left(\iota^{\uparrow}, \iota^{\uparrow}\right)$-continuous. It also holds whenever $g^{\sigma} f^{\sigma}(\sigma, \iota)$ continuous, and hence in particular when either $f^{\sigma}$ is $(\sigma, \sigma)$-continuous and $g^{\sigma}$ is $(\sigma, \iota)$-continuous or $f^{\sigma}$ is $(\sigma, \iota)$-continuous and $g^{\sigma}$ is $(\iota, \iota)$-continuous.

Theorem 2.33. For any isotone DL maps $f: \mathbf{A} \rightarrow \mathbf{B}$ and $g: \mathbf{B} \rightarrow \mathbf{C}$, the equality $(g f)^{\sigma}=g^{\sigma} f^{\sigma}$ holds if $g^{\sigma}$ is upper continuous, hence in particular if $g$ is an operator.

Proof. By Theorem 2.30.

EXAMPLE 2.34. The inequality in part (i) of Theorem 2.30 may be strict.

Let $\mathbf{B}$ be a bounded chain that is dense in itself, with $\mathbf{A}$ a dense subchain of $\mathbf{B}$, containing 0 and 1 , such that $B-A$ is also a dense subset of $B$. Let $f: \mathbf{A} \rightarrow \mathbf{B}$ be the inclusion map, and $g: \mathbf{B} \rightarrow \mathbf{B}$ the map that sends each element in $A$ to 1 and each element in $B-A$ to 0 . Then $(g f)(x)=1$ for all $x \in A$, and hence $(g f)^{\sigma}(x)=1$ for all $x \in A^{\sigma}$. On the other hand, $g^{\sigma} f^{\sigma}(x)=0$ for all $x \in A^{\sigma}-A$. This will be proved by showing that the following two claims hold.

Claim 1. $f^{\sigma}$ sends $A^{\sigma}-A$ into $B^{\sigma}-B$.

Claim 2. $g^{\sigma}$ sends $B^{\sigma}-B$ into 0 . 
In proving the first claim, we use the fact that $f$ preserves both joins and meets, and is therefore $(\sigma, \sigma)$-continuous. For $b \in B$, the set $\left(f^{\sigma}\right)^{-1}(b)$ is therefore open and has a dense subset consisting of elements of $A$. But $\left(f^{\sigma}\right)^{-1}(b)$ contains at most one member of $A$; therefore it contains no member of $A^{\sigma}-A$.

The second claim follows from the observation that if $y \in B^{\sigma}-B$, then every basic $\sigma$-neighborhood $U$ of $y$ contains at least two members of $B$, and therefore contains a member of $B-A$, so that $\bigwedge g(U \cap B)=0$.

EXAMPLE 2.35. The inequality in part (ii) of Theorem 2.30 may be strict.

This example combines the operation of Example 2.26 and the dual of the operation in Example 2.27. Let $f: \mathbf{B} \times \mathbf{B} \rightarrow \mathbf{B}$ be the lattice join, and let $g: \mathbf{B} \rightarrow \mathbf{B}$ be the map such that $g(1)=1$ and $g(z)=0$ for $0 \neq z \in B$. Then, for $x, y \in B^{\sigma}, g^{\sigma} f^{\sigma}(x, y)=1$ if and only if $f^{\sigma}(x, y)=1$, if and only if $x \vee y=1$. On the other hand, $(g f)^{\sigma}(x, y)=1$ if and only if there exist closed elements $p \leq x$ and $q \leq y$ such that $p \vee q=1$. Hence, if we take $\mathbf{B}$ to be an infinite Boolean algebra, and take $x$ and $y$ to be complementary elements of $\mathbf{B}^{\sigma}$ that are not in $\mathbf{B}$, then $(g f)^{\sigma}(x, y)=0<1=g^{\sigma} f^{\sigma}(x, y)$.

\section{Preservation of homomorphisms}

\subsection{Canonical extensions of homomorphisms}

Definition 3.1. For a DLE $\mathbf{A}=\left(\mathbf{A}_{0}, \omega^{\mathbf{A}}, \omega \in \Omega\right)$ we define the canonical extension of $\mathbf{A}$ and the dual canonical extension of $\mathbf{A}$ to be the DLEs

$$
\begin{aligned}
& \mathbf{A}^{\sigma}=\left(\mathbf{A}_{0}^{\sigma},\left(\omega^{\mathbf{A}}\right)^{\sigma}, \omega \in \Omega\right), \\
& \mathbf{A}^{\pi}=\left(\mathbf{A}_{0}^{\sigma},\left(\omega^{\mathbf{A}}\right)^{\pi}, \omega \in \Omega\right),
\end{aligned}
$$

respectively.

Observe that if we define the dual of a DLE $\mathbf{A}=\left(\mathbf{A}_{0}, \omega^{\mathbf{A}}, \sigma \in \Omega\right)$ to be the $\operatorname{DLE} \mathbf{A}^{\alpha}=\left(\mathbf{A}_{0}^{\alpha}, \omega^{\mathbf{A}}, \sigma \in \Omega\right)$, then $\mathbf{A}^{\pi}=\mathbf{A}^{\alpha \sigma \alpha}$.

Many properties are not preserved by canonical extensions. A striking case will be provided in Example 3.8: a DLE homomorphism $h: \mathbf{A} \rightarrow \mathbf{B}$ whose canonical extension $h: \mathbf{A} \rightarrow \mathbf{B}$ is not a homomorphism. It is the objective of this section to show that, under some quite general conditions, homomorphisms are preserved.

Every DL homomorphism $h: \mathbf{A} \rightarrow \mathbf{B}$ is preserved by canonical extensions. Even more is true, $h^{\sigma}: \mathbf{A}^{\sigma} \rightarrow \mathbf{B}^{\sigma}$ is always a complete homomorphism. In fact, $h^{\sigma}$ is smooth by Corollary 2.25, and using Theorem 2.21 and its dual we infer that $h^{\sigma}$ is completely join and meet preserving. The maps $\mathbf{A} \mapsto \mathbf{A}^{\sigma}$ and 
$h \mapsto h^{\sigma}$ therefore form a functor from the category of all DLs and their homomorphisms into the category of all $\mathrm{DL}^{+} \mathrm{s}$ and their complete homomorphisms. We need some more information about this functor.

THEOREM 3.2. The maps $\mathbf{A} \longmapsto \mathbf{A}^{\sigma}$ and $h \longmapsto h^{\sigma}$ form a functor from the category of all DLs and their homomorphisms into the category of all $\mathrm{DL}^{+} s$ and their complete homomorphisms. This functor preserves injectivity and surjectivity of homomorphisms.

Proof. We have just observed that homomorphisms go into complete homomorphisms, and we know that the identity homomorphism from $\mathbf{A}$ to $\mathbf{A}$ is sent into the identity homomorphism from $\mathbf{A}^{\sigma}$ to $\mathbf{A}^{\sigma}$. Furthermore, if $h: \mathbf{A} \rightarrow \mathbf{B}$ and $h^{\prime}: \mathbf{B} \rightarrow \mathbf{C}$ are DL homomorphism, then $h^{\prime \sigma} h^{\sigma}=\left(h^{\prime} h\right)^{\sigma}$. This shows that the maps form a functor.

Now we prove that $\sigma$ preserves surjectivity and injectivity of homomorphisms. If the DL homomorphism $h: \mathbf{A} \rightarrow \mathbf{B}$ is surjective, then the range $S$ of $h^{\sigma}$ contains $B$, and since $S$ is closed under arbitrary joins and meets, it follows that $S=B^{\sigma}$. That is, $h^{\sigma}$ is surjective. Finally we assume that $h$ is injective, and show that $h^{\sigma}$ is also injective. It suffices to show that, for $x, y \in A^{\sigma}$, $h^{\sigma}(x) \leq h^{\sigma}(y)$ implies $x \leq y$. In fact, since $h^{\sigma}$ is completely join and meet preserving, we only need to prove this implication for the special case when $x \in J^{\infty}\left(\mathbf{A}^{\sigma}\right)$ and $y \in M^{\infty}\left(\mathbf{A}^{\sigma}\right)$. In this case, $h^{\sigma}(x)=\bigwedge h(\uparrow x \cap A)$ and $h^{\sigma}(y)=\bigvee h(\downarrow y \cap A)$, so that $\bigwedge h(\uparrow x \cap A) \leq \bigvee h(\downarrow y \cap A)$. Using the compactness property and the fact that the set $h(\uparrow x \cap A)$ is down-directed and $h(\downarrow y \cap A)$ is up-directed, we conclude that there exist $a, b \in A$ such that $x \leq a, b \leq y$ and $h(a) \leq h(b)$. Since $h$ is injective, the last inclusion implies that $a \leq b$, and hence $x \leq y$.

The assertion that a DLE map $h: \mathbf{A} \rightarrow \mathbf{B}$ is a homomorphism means that, for corresponding basic operations $f$ of $\mathbf{A}$ and $g$ of $\mathbf{B}$, say of arity $n$, the equation $h f=g h^{[n]}$ holds, and to say that the induced map $h^{\sigma}: \mathbf{A}^{\sigma} \rightarrow \mathbf{B}^{\sigma}$ is a homomorphism means that the equation $h^{\sigma} f^{\sigma}=g^{\sigma} h^{\sigma[n]}$ holds. In order to show that the first equation implies the second, one might try to prove the string of equalities

$$
h^{\sigma} f^{\sigma}=(h f)^{\sigma}=\left(g h^{[n]}\right)^{\sigma}=g^{\sigma} h^{[n] \sigma}=g^{\sigma} h^{\sigma[n]} .
$$

The second equality obviously holds and the fourth is easy to verify. It will be shown that the first equality also holds, as does the inequality $g^{\sigma} h^{[n] \sigma} \leq$ $\left(g h^{[n]}\right)^{\sigma}$. The problem of determining which homomorphisms are preserved by canonical extensions therefore reduces to finding out under what conditions the inequality $\left(g h^{[n]}\right)^{\sigma} \leq g^{\sigma} h^{[n] \sigma}$ holds. 
Lemma 3.3. For any DLE maps $f: \mathbf{A} \rightarrow \mathbf{B}$ and $h: \mathbf{B} \rightarrow \mathbf{C}$, if $h$ is join and meet preserving, then $h^{\sigma} f^{\sigma}=(h f)^{\sigma}$.

Proof. Using the fact that $h^{\sigma}$ preserves all non-empty joins and meets, we compute

$$
\begin{aligned}
h^{\sigma} f^{\sigma}(x) & =h^{\sigma}\left(\bigvee\left\{\bigwedge f(U \cap A): x \in U \in \sigma\left(\mathbf{A}^{\sigma}\right)\right\}\right) \\
& =\bigvee\left\{\bigwedge h f(U \cap A): x \in U \in \sigma\left(\mathbf{A}^{\sigma}\right)\right\}=(h f)^{\sigma}(x) .
\end{aligned}
$$

Lemma 3.4. For any DL maps $h: \mathbf{A} \rightarrow \mathbf{B}$ and $g: \mathbf{B} \rightarrow \mathbf{C}$, if $h$ is join and meet preserving, then $g^{\sigma} h^{\sigma} \leq(g h)^{\sigma}$.

Proof. By Theorem 2.24(iv), $h^{\sigma}$ is $(\sigma, \sigma)$-continuous, Hence $g^{\sigma} h^{\sigma} \leq$ $(g h)^{\sigma}$ by Corollary 2.31(i).

Theorem 3.5. For any DLE homomorphism $h: \mathbf{A} \rightarrow \mathbf{B}$, the map $h^{\sigma}:$ $\mathbf{A}^{\sigma} \rightarrow \mathbf{B}^{\sigma}$ is a homomorphism if and only if, for every auxiliary operation $g$ of $\mathbf{B},\left(g h^{[n]}\right)^{\sigma} \leq g^{\sigma} h^{[n] \sigma}$, where $n$ is the arity of $g$.

Proof. By the preceding two lemmas.

Lemma 3.6. For any DL maps $h: \mathbf{A} \rightarrow \mathbf{B}$ and $g: \mathbf{B} \rightarrow \mathbf{C}$, with $h$ join and meet preserving, if $h$ is surjective, then $(g h)^{\sigma}=g^{\sigma} h^{\sigma}$.

Proof. For $x \in A^{\sigma}$ we have

$$
\begin{aligned}
& (g h)^{\sigma}(x)=\bigvee\left\{\bigwedge g h(U \cap A): x \in U \in \sigma\left(\mathbf{A}^{\sigma}\right)\right\}, \\
& g^{\sigma} h^{\sigma}(x)=\bigvee\left\{\bigwedge g(V \cap B): h^{\sigma}(x) \in V \in \sigma\left(\mathbf{B}^{\sigma}\right)\right\} .
\end{aligned}
$$

It will be shown that each of the joinands $\bigwedge g h(U \cap A)$ in the first formula is equal to the joinand $\bigwedge g(V \cap B)$ in the second with $V=h^{\sigma}(U)$. First we need to show that $h^{\sigma}$ sends open sets into open sets. It is sufficient to do this for the case of a basic open set $U=[p, u]$. From the fact that $h$ is isotone, it follows that $h^{\sigma}(U) \subseteq\left[h^{\sigma}(p), h^{\sigma}(u)\right]$. To prove that equality holds, consider any $y \in\left[h^{\sigma}(p), h^{\sigma}(u)\right]$. By Theorem 3.2, $y=h^{\sigma}(x)$ for some $x \in A^{\sigma}$, and hence $y=h^{\sigma}((x \wedge u) \vee p) \in h^{\sigma}(U)$.

Our set $V=h^{\sigma}(U)$ is therefore open. We next show that

$$
h(U \cap A)=V \cap B
$$

Obviously, $h(U \cap A) \subseteq h^{\sigma}(U) \cap h(A)=V \cap B$. On the other hand, if $b \in V \cap B$, then $b$ is an isolated point, and the set $U_{0}=\left(h^{\sigma}\right)^{-1}(b)$ is therefore 
open. Also, $b=h^{\sigma}(x)$ for some $x \in U$, so that $U$ and $U_{0}$ are not disjoint. In other words, the open set $U \cap U_{0}$ is not empty, and therefore contains a point $a \in A$. Therefore, $b=h(a) \in h(U \cap A)$. This completes the proof of (3.1), and it readily follows that $\bigwedge g h(U \cap A)=\bigwedge g(V \cap B)$.

Thus $(g h)^{\sigma} \leq g^{\sigma} h^{\sigma}$. The opposite inequality holds without the assumption that $h$ be surjective.

THEOREM 3.7. Every surjective DLE homomorphism is preserved by canonical extensions.

Proof. By the preceding theorem.

At this point one may wonder whether homomorphisms are always preserved by canonical extensions. Since surjective ones always are, and since any homomorphism splits into a surjective, and an injective one, there must be an injective counterexample if there is one at all.

Example 3.8. This is an example of a DL $\mathbf{B}=\left(\mathbf{B}_{0}, g\right)$ and a subalgebra $\mathbf{A}=\left(\mathbf{A}_{0}, f\right)$ such that $g^{\sigma} h^{[n] \sigma}<\left(g h^{[n]}\right)^{\sigma}$, where $h: A \rightarrow B$ is the injection homomorphism and $n$ is the arity of $g$.

Essentially, this algebra was already constructed in Example 2.34, except that we need to do some renaming: We denote by $\mathbf{B}_{0}$ the DL called $\mathbf{B}$ there. Set $\mathbf{B}=\left(\mathbf{B}_{0}, g\right)$, and let $\mathbf{A}=\left(\mathbf{A}_{0}, f\right)$ be the subalgebra of $\mathbf{B}$ with universe $A$. The injective homomorphism $h$ is then the map previously called $f$. As the calculations carried out there show, $g^{\sigma} h^{\sigma}<(g h)^{\sigma}$.

\subsection{The property $(\mathrm{PH})$}

The fact that surjective homomorphisms always are preserved by canonical extension, and thus that preservation of homomorphisms, when it goes wrong, goes wrong also for an injective homomorphism with the same codomain, allows us to consider preservation of homomorphisms as a property of the codomain (and thus of a single algebra) rather than as a property of maps. We make the following definition:

DEFINITION 3.9.

(i) A DLE $\mathbf{B}$ is said to have the property $(\mathrm{PH})$ if every homomorphism $h: \mathbf{A} \rightarrow \mathbf{B}$ from a DLE $\mathbf{A}$ into $\mathbf{B}$ is preserved by homomorphisms.

(ii) A class $\mathscr{A}$ of DLEs is said to have the property (PH) if every member of $\mathscr{K}$ has this property.

We make the comment that precedes this definition precise:

THEOREM 3.10. A DLE $\mathbf{B}$ has the property $(\mathrm{PH})$ if and only if every injective homomorphism $h: \mathbf{A} \rightarrow \mathbf{B}$ is preserved by canonical extension. 
Proof. An arbitrary homomorphism $h: \mathbf{A} \rightarrow \mathbf{B}$ can be factored into a surjective homomorphism $h^{\prime}: \mathbf{A} \rightarrow \mathbf{A}^{\prime}$ onto the subalgebra $\mathbf{A}^{\prime}=h(\mathbf{A})$ of $\mathbf{B}$ and the injection $h^{\prime \prime}: \mathbf{A}^{\prime} \rightarrow \mathbf{B}$. Given an $n$-ary basic operation $g$ of $\mathbf{B}$, we apply Lemma 3.6 to the maps $h^{\prime[n]}: \mathbf{A}^{n} \rightarrow \mathbf{A}^{\prime n}$ and $g h^{\prime \prime} \mathbf{A}^{\prime n} \rightarrow \mathbf{B}$ to infer that $\left(g h^{[n]}\right)^{\sigma}=\left(g h^{\prime \prime[n]}\right)^{\sigma} h^{\prime[n] \sigma}$. Since we are assuming that $\left(g h^{\prime \prime[n]}\right)^{\sigma} \leq g^{\sigma} h^{\prime \prime[n] \sigma}$, this yields $\left(g h^{[n]}\right)^{\sigma} \leq g^{\sigma} h^{\prime \prime[n] \sigma} h^{\prime n] \sigma}=g^{\sigma} h^{[n] \sigma}$.

Our notation contains an ambiguity that is harmless in most situations, but can be misleading. A DLE homomorphism $h: \mathbf{A} \rightarrow \mathbf{B}$ may also be regarded as a homomorphism $h: \mathbf{A} \rightarrow \mathbf{C}$, where $\mathbf{C}$ is an extension of $\mathbf{B}$. However, the canonical extension $h^{\sigma}$ depends not only on the map $h$ but also on the source algebra and the target algebra. In fact, it may happen that $h^{\sigma}: \mathbf{A}^{\sigma} \rightarrow \mathbf{B}^{\sigma}$ is a homomorphism but $h^{\sigma}: \mathbf{A}^{\sigma} \rightarrow \mathbf{C}^{\sigma}$ is not. The next theorem says essentially that if the second map is a homomorphism, then so is the first.

A DLE of similarity type $\mu$ will be referred to as a $\operatorname{DLE}_{\mu}$.

THEOREM 3.11. Let $\mathfrak{\Re}$ be the class of all $\mathrm{DLE}_{\mu} s$ with the property (PH). Then $\mathrm{S}(\Re)=\Re$.

Proof. Consider injective homomorphisms

$$
\mathbf{A} \stackrel{h}{\longrightarrow} \mathbf{B}^{\prime} \stackrel{g}{\longrightarrow} \mathbf{B} .
$$

If $\mathbf{B}$ has the property (PH), then $g^{\sigma}$ and $(g h)^{\sigma}=g^{\sigma} h^{\sigma}$ are homomorphisms. Since $g^{\sigma}$ is injective, it follows that $h^{\sigma}$ is a homomorphism.

TheOREM 3.12. Let $\AA$ be the class of all $\mathrm{DLE}_{\mu} s$ with the property $(\mathrm{PH})$. Then $\mathrm{H}(\Re)=\Re$.

Proof. Suppose $h_{b}: \mathbf{B}_{1} \rightarrow \mathbf{B}$ is a surjective DLE homomorphism and $\mathbf{B}_{1}$ has the property $(\mathrm{PH})$. We need to show that $\mathbf{B}$ also has the property (PH). That is, given an injective homomorphism $h: \mathbf{A} \rightarrow \mathbf{B}$, we need to show that $h^{\sigma}: \mathbf{A}^{\sigma} \rightarrow \mathbf{B}^{\sigma}$ is also a homomorphism. Let $\mathbf{A}_{1}=h_{b}^{-1} h(\mathbf{A})$, and let $h_{1}: \mathbf{A}_{1} \rightarrow \mathbf{B}_{1}$ be the injective homomorphism of the subalgebra $\mathbf{A}_{1}$ of $\mathbf{B}_{1}$ into $\mathbf{B}_{1}$. Also let $h_{a}: \mathbf{A}_{1} \rightarrow \mathbf{A}$ be the unique map that makes the diagram

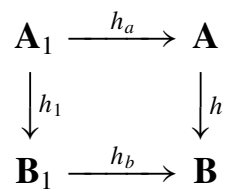

commute. Then $h_{a}$ is a surjective homomorphism. Noting that $\left(h h_{a}\right)^{\sigma}=h^{\sigma} h_{a}^{\sigma}$ and $\left(h_{b} h_{1}\right)^{\sigma}=h_{b}^{\sigma} h_{1}^{\sigma}$, because $h$ and $h_{b}$ are join and meet preserving, we infer 
that the diagram

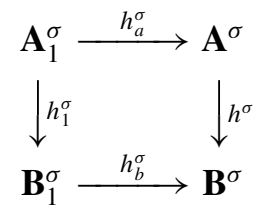

also commutes. Now $h_{a}^{\sigma}$ and $h_{b}^{\sigma}$ are homomorphisms because $h_{a}$ and $h_{b}$ are surjective, and $h_{1}^{\sigma}$ is a homomorphism because $\mathbf{B}_{1}$ has the property (PH). Thus $h^{\sigma} h_{a}^{\sigma}=h_{b}^{\sigma} h_{1}^{\sigma}$ is a homomorphism. Since $h_{a}^{\sigma}$ is a surjective homomorphism, it follows that $h^{\sigma}$ is a homomorphism.

So the class of all $\mathrm{DLE}_{\mu} \mathrm{s}$ with the property $(\mathrm{PH})$ is closed both under the formation of homomorphic images and subalgebras. It is now natural to wonder whether it is closed under the formation of direct products.

Example 3.13. The direct product of finite $\mathrm{DLE}_{\mu} \mathrm{s}$ need not have the property $(\mathrm{PH})$.

Let $\mathbf{B}=\left(\mathbf{B}_{0}, g\right)$ be as in Example 3.8. This algebra is locally finite; indeed, every subset of $B$ containing the elements 0 and 1 is the universe of a subalgebra. Therefore $\mathbf{B}$ is isomorphic to a homomorphic image of a subalgebra of the direct product $\mathbf{B}^{\prime}$ of all the finite subalgebras of $\mathbf{B}$. Since $\mathbf{B}$ does not have the property $(\mathrm{PH})$, neither does $\mathbf{B}^{\prime}$. Finally, finite algebras clearly have (PH), as for an injective homomorphism $h: \mathbf{A} \rightarrow \mathbf{B}$ with $\mathbf{B}$ finite, $h^{\sigma}=h$.

\subsection{Boolean products and $(\mathrm{PH})$}

As already noted, the canonical extension of the direct product of finitely many DLEs is (or can be identified with) the direct product of the canonical extensions of the factors. This is not true for products with infinitely many factors. In fact, the proper generalization of this result is that the canonical extension of a Boolean product of DLEs is the direct product of the canonical extensions of the factors. To apply this to direct products, we must therefore represent the direct product as a Boolean product by adding more factors, and then take canonical extensions of the factors.

We begin by recalling some basic facts about Boolean products. For a more comprehensive treatment see [4].

Definition 3.14. By a weak Boolean product of algebras $\mathbf{C}_{x}, x \in X$, where $X$ is a Boolean space, we mean a subdirect product $\mathbf{B}$ of the algebras $\mathrm{C}_{x}$ such that

(i) For all $a, b \in B$, the set $[a=b]=\{x \in X: a(x)=b(x)\}$ is open;

(ii) (The Patching Property) For all $a, b \in B$ and $Y \subseteq X$ clopen, we have $(a \uparrow Y) \cup(b \uparrow(X-Y)) \in B$; 
(iii) The set $\left\{x \in X:\left|C_{x}\right|>1\right\}$ is dense in $X$.

If, in place of the first condition, the stronger condition

(i') For all $a, b \in B$, the set $[a=b]=\{x \in X: a(x)=b(x)\}$ is clopen;

holds, then $\mathbf{B}$ is said to be a Boolean product of the algebras $\mathbf{C}_{x}$.

An isomorphism from an algebra $\mathbf{B}$ onto a (weak) Boolean product is called a (weak) Boolean decomposition of $\mathbf{B}$.

The following theorem is an algebraic version of the corresponding theorem for Stone spaces given in [6].

THEOREM 3.15. Suppose

$$
g: \mathbf{B} \rightarrow \Pi_{x \in X} \mathbf{C}_{x}
$$

is a weak Boolean decomposition of the DLE B. If all the induced homomorphisms $g_{x}: \mathbf{B} \rightarrow \mathbf{C}_{x}$ are preserved by canonical extensions, then

$$
g^{\prime}: \mathbf{B}^{\sigma} \simeq \Pi_{x \in X} \mathbf{C}_{x}^{\sigma}
$$

where, for all $b \in B$ and $x \in X, g^{\prime}(b)(x)=g_{x}^{\sigma}(b)$.

Proof. By hypothesis, the maps $g_{x}^{\sigma}: \mathbf{B}^{\sigma} \rightarrow \mathbf{C}_{x}^{\sigma}$ are homomorphisms, and the induced map $g^{\prime}$ is therefore a homomorphism. Also, since the homomorphisms $g_{x}^{\sigma}$ are complete, so is $g^{\prime}$. To complete the proof, we have to show that $g^{\prime}$ is bijective. This will not involve the auxiliary operations, so we may assume that the algebras are DLs.

Let $\mathbf{C}=g(\mathbf{B})$ and $\mathbf{C}^{\prime}=\Pi_{x \in X} \mathbf{C}_{x}^{\sigma}$. From Theorem 2.1(iii) we see that $\mathbf{C}^{\prime}$ is a $\mathrm{DL}^{+}$.

Claim 1. C is a separating sublattice of $\mathbf{C}^{\prime}$. The members of $J^{\infty}\left(\mathbf{C}^{\prime}\right)$ are the elements $p \in C^{\prime}$ such that, for some $x \in X, p_{x} \in J^{\infty}\left(\mathbf{C}_{x}^{\sigma}\right)$ while $p_{y}=0$ for all other $y \in X$. Consider $p, q \in J^{\infty}\left(\mathbf{C}^{\prime}\right)$ with $p \not \leq q$, and let $x$ and $y$ be the members of $X$ with $p_{x} \neq 0$ and $q_{y} \neq 0$. We need an element $c \in C$ with $q \leq c$ and $p \not \leq c$. If $x=y$, then we pick an element $d \in B_{x}$ with $q_{x} \leq d$ and $p_{x} \not \leq d$, and using the fact that $\mathbf{C}$ is a subdirect product of the algebras $\mathbf{C}_{x}$, take $c$ to be a member of $C$ with $c_{x}=d$. If $x \neq y$, then we pick a clopen subset $Y$ of $X$ that contains $y$ as a member, but not $x$, and using the patching property, take $c$ to be the member of $C$ with $c_{z}=1$ for $z \in Y$ and $c_{z}=0$ for $z \in X-Y$.

Claim 2. C is compact in $\mathbf{C}^{\prime}$. Consider sets $S, T \subseteq C$ with $\bigwedge S \leq \bigvee T$ (in $\left.\mathbf{C}^{\prime}\right)$. For each $x \in X$ we then have $(\bigwedge S)_{x} \leq(\bigvee T)_{x}$, and hence $\left(\bigwedge F_{x}\right)_{x} \leq$ $\left(\bigvee G_{x}\right)_{x}$ for some finite sets $F_{x} \subseteq S$ and $G_{x} \subseteq T$. For $x \in X$ let $N_{x}=\{y \in$ $\left.X:\left(\bigwedge F_{x}\right)_{y} \leq\left(\bigvee G_{x}\right)_{y}\right\}$. These sets are open, and they cover $X$. Hence $X$ is covered by some finite sequence of sets $N_{x_{i}}, i=0,1, \ldots, n-1$. Taking $F$ to 
be the union of the sets $F_{x_{i}}$ and $G$ to be the union of the sets $G_{x_{i}}$, we easily see that $\bigwedge F \leq \bigvee G$.

Having shown that $\mathbf{C}$ is a separating and compact sublattice of $\mathbf{C}^{\prime}$, we conclude by Theorem 2.5 that $g$ can be extended to an isomorphism $g^{\prime \prime}: \mathbf{B}_{0}^{\sigma} \simeq$ $\mathbf{C}_{0}^{\prime}$ of the underlying lattices.

Returning to the general case, we have on the one hand a complete homomorphism $g^{\prime}: \mathbf{B}^{\sigma} \rightarrow \mathbf{C}^{\prime}$, and on the other hand an isomorphism $g^{\prime \prime}: \mathbf{B}_{0}^{\sigma} \simeq \mathbf{C}_{0}^{\prime}$. From the fact that the two maps agree on $B$, and that both of them preserve all joins and meets, it follows that the underlying set maps are in fact equal. Thus $g^{\prime}: \mathbf{B}^{\sigma} \simeq \mathbf{C}^{\prime}$.

TheOREM 3.16. The class of all $\mathrm{DLE}_{\mu} s$ with the property (PH) is closed under the formation of weak Boolean products.

Proof. Consider a DLE $\mathbf{B}$ that has a weak Boolean decomposition $g$ : $\mathbf{B} \rightarrow \Pi_{x \in X} \mathbf{C}_{x}$ such that all the factors $\mathbf{C}_{x}$ have the property (PH). Given an injective homomorphism $h: \mathbf{A} \rightarrow \mathbf{B}$, we need to show that $h^{\sigma}: \mathbf{A}^{\sigma} \rightarrow \mathbf{B}^{\sigma}$ is a homomorphism.

In the notation of the preceding theorem, $g^{\prime}: \mathbf{B}^{\sigma} \simeq \mathbf{C}^{\prime}$. Also, from the fact that the algebras $\mathbf{C}_{x}$ have the property $(\mathrm{PH})$ it follows that the homomorphisms $g_{x} h: \mathbf{A} \rightarrow \mathbf{C}_{x}$ are preserved by canonical extensions. That is, $\left(g_{x} h\right)^{\sigma}=$ $g_{x}^{\sigma} h^{\sigma}: \mathbf{A}^{\sigma} \rightarrow \mathbf{C}_{x}^{\sigma}$ is a homomorphism for every $x \in X$. Consequently, the induced map $g^{\prime \prime}: \mathbf{A}^{\sigma} \rightarrow \mathbf{C}^{\prime}$ is a homomorphism, but this is just the map $g^{\prime} h^{\sigma}$. Since $g^{\prime}$ is an isomorphism, we conclude that $h^{\sigma}$ is a homomorphism.

\subsection{Direct products and $(\mathrm{PH})$}

The theorem about canonical extensions of Boolean products will now be used to describe canonical extensions of direct products.

THEOREM 3.17. Given a direct decomposition

$$
h: \mathbf{A} \simeq \prod_{i \in I} \mathbf{B}_{i}
$$

of an algebra $\mathbf{A}$, with each factor $\mathbf{B}_{i}$ nontrivial, let $X$ be the Boolean space of all ultrafilters on I, and for $x \in X$ let $\mathbf{C}_{x}=\prod_{i \in I} \mathbf{B}_{i} / x$. For $a \in A$ and $x \in X$, let $h^{\prime}(a)(x)=h(a) / x$. Then the map

$$
h^{\prime}: \mathbf{A} \rightarrow \Pi_{x \in X} \mathbf{C}_{x}
$$

is a Boolean decomposition of $\mathbf{A}$.

Proof. It is easy to see that $h^{\prime}$ is an injective homomorphism. We show that $h^{\prime}(\mathbf{A})$ is a Boolean product. For $a, b \in A$,

$$
\left[h^{\prime}(a)=h^{\prime}(b)\right]=\{x \in X:[h(a)=h(b)] \in x\},
$$


which is a clopen subset of $X$.

In proving the patching property, we use the fact that the principal ultrafilters are isolated points of $X$, and that they form a dense subset of $X$. Given $a, b \in$ $A$, and a clopen subset $Y$ of $X$, let $J$ be the set of all $i \in I$ such that the corresponding ultrafilter $x(i)=\{J \subseteq I: i \in J\}$ is in $Y$, and let $c$ be the member of $A$ such that $h(c)$ agrees with $h(a)$ on $J$ and with $h(b)$ on $I-J$. Then $h^{\prime}(c)$ agrees with $h^{\prime}(a)$ on a dense subset of $Y$, and the two therefore agree on $Y$. Similarly, $h^{\prime}(c)$ agrees with $h^{\prime}(b)$ on $X-Y$. Finally, since the factors $\mathbf{B}_{i}$ are non-trivial, so are the ultraproducts $\mathbf{C}_{x}$. Thus $h^{\prime}$ is a Boolean decomposition of $\mathbf{A}$.

Usually, the details of the construction of the Boolean decomposition are not needed. We therefore give an abbreviated version of the last theorem.

Theorem 3.18. If the algebra $\mathbf{A}$ has a direct decomposition

$$
\mathbf{A} \simeq \prod_{i \in I} \mathbf{A}_{i} .
$$

then $\mathbf{A}$ has a Boolean decomposition

$$
\mathbf{A} \rightarrow \Pi_{x \in X} \mathbf{B}_{x},
$$

where each $\mathbf{B}_{x}$ is an ultraproduct of the algebras $\mathbf{A}_{i}$.

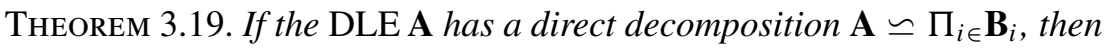
$\mathbf{A}^{\sigma}$ has a direct decomposition $\mathbf{A}^{\sigma} \simeq \Pi_{x \in X} \mathbf{C}_{x}^{\sigma}$, where each of the factors $\mathbf{C}_{x}$ is an ultraproduct of the algebras $\mathbf{B}_{i}$.

THEOREM 3.20. The direct product of $\operatorname{DLE}_{\mu} s \mathbf{B}_{i}, i \in I$, has the property $(\mathrm{PH})$ if and only if every ultraproduct $\Pi_{i \in I} \mathbf{B}_{i} / x$ has the property $(\mathrm{PH})$.

Theorem 3.21. For any class $\Re$ of $\operatorname{DLE}_{\mu} s$, if $\mathrm{P} u(\Re)$ has the property $(\mathrm{PH})$, then so does $\mathrm{V}(\Re)$.

Proof. If $\mathrm{P} u(\Re)$ has the property $(\mathrm{PH})$, then by the preceding theorem, so does $\mathrm{P}(\Re)$, and by Theorems 3.11 and 3.12, the same is true of $\mathrm{V}(\Re)=$ $\operatorname{HSP}(\Re)$.

\subsection{Some varieties with the property $(\mathrm{PH})$}

Using Theorem 3.21, we can give numerous examples of varieties having the property $(\mathrm{PH})$.

THEOREM 3.22. Every finitely generated variety of $\mathrm{DLE}_{\mu}$ s has the property $(\mathrm{PH})$. 
Proof. If $\Re$ is a finite set of finite $\operatorname{DLE}_{\mu} \mathrm{s}$, then every ultraproduct of members of $\Re$ is (isomorphic to) a member of $\mathscr{R}$.

DEFINITION 3.23.

(i) A DL operation $f: \mathbf{B}_{0} \times \mathbf{B}_{1} \times \cdots \times \mathbf{B}_{n-1} \rightarrow \mathbf{C}$ is said to be monotone if, for each $i<n$, the functions obtained by fixing all its arguments except the $i$ th one are either all isotone or all antitone.

(ii) $\mathrm{A}$ DLE $\mathbf{A}=\left(\mathbf{A}_{0}, \omega^{\mathbf{A}}, \omega \in \Omega\right)$ is said to be monotone (or to be a DLM) if each of the operations $\omega^{\mathbf{A}}$ is monotone.

(iii) $\mathrm{A}$ DLE $\mathbf{A}=\left(\mathbf{A}_{0}, \omega^{\mathbf{A}}, \omega \in \Omega\right)$ is said to be smooth (or to be a DLS) if each of the operations $\omega^{\mathbf{A}}$ is smooth.

A monotone DL operation $f: \mathbf{B}_{0} \times \mathbf{B}_{1} \times \cdots \times \mathbf{B}_{n-1} \rightarrow \mathbf{C}$ may be regarded as an isotone operation by replacing some of the factors $\mathbf{B}_{i}$ by their duals $\mathbf{B}_{i}^{\alpha}$. In greater detail, we treat $f$ as an isotone map $f: \mathbf{B}_{0}^{\varepsilon(0)} \times \mathbf{B}_{1}^{\varepsilon(1)} \times \cdots \times \mathbf{B}_{n-1}^{\varepsilon(n-1)} \rightarrow$ $\mathbf{C}$, where for each $i<n, \varepsilon(i)$ is either 1 or $\alpha$, depending on whether $f$ is isotone or antitone as a function of its $i$ th coordinate. This sequence $\varepsilon$ will be referred to as the monotonicity type of $f$. For brevity we sometimes write $\mathbf{B}^{\varepsilon}$ for $\mathbf{B}_{0}^{\varepsilon(0)} \times \mathbf{B}_{1}^{\varepsilon(1)} \times \cdots \times \mathbf{B}_{n-1}^{\varepsilon(n-1)}$. It is important to note that this change of perspective does not change the canonical extension. This is so because, for a DL $\mathbf{B}$, the topologies $\sigma\left(\mathbf{B}^{\sigma}\right)$ and $\sigma\left(\mathbf{B}^{\alpha \sigma}\right)$ are identical.

LEMMA 3.24. Every DLM has the property (PH).

Proof. Suppose B is a DLM. As observed above, each of the basic operations $g$ of arity $n$ may be regarded as an isotone map $g: \mathbf{B}^{\varepsilon} \rightarrow \mathbf{B}$, where $\varepsilon$ is the monotonicity type of $g$. Hence, for any DLE homomorphism $h: \mathbf{A} \rightarrow \mathbf{B}$, $\left(g h^{[n]}\right)^{\sigma} \leq g^{\sigma} h^{[n] \sigma}$ by Theorem 2.28. Therefore $\mathbf{B}$ has the property (PH) by Theorem 3.10.

THEOREM 3.25. The variety generated by the class of all $\mathrm{DLM}_{\mu} s$ has the property $(\mathrm{PH})$.

Proof. Let $\Re$ be the class of all $\operatorname{DLM}_{\mu} \mathrm{s} \mathbf{B}=\left(\mathbf{B}_{0}, \omega \mathbf{B}, \omega \in \Omega\right)$. By the preceding lemma, $\Re$ has the property (PH). Fixing $\omega \in \Omega$, we can partition $\AA$ into finitely many classes $\Re_{i}$ in such a way that, for two members $\mathbf{B}$ and $\mathbf{B}^{\prime}$ of the same class $\Re_{i}$, the operations $\omega^{\mathbf{B}}$ and $\omega^{\mathbf{B}^{\prime}}$ have the same monotonicity type. From this it follows that, for any ultraproduct $\mathbf{B}$ of members of $\Re$, the operation $\omega^{\mathbf{B}}$ is monotone. Since this is true for every $\omega \in \Omega$, we infer that $\Omega$ is closed under ultraproducts. Hence by Theorem 3.21, $\mathrm{V}(\Re)$ has the property (PH).

THEOREM 3.26. The class of all DLSs has the property (PH). 
Proof. Given a DLS-homomorphism $h: \mathbf{A} \rightarrow \mathbf{B}$, we need to show that $h^{\sigma}: \mathbf{A}^{\sigma} \rightarrow \mathbf{B}^{\sigma}$ is also a homomorphism. Let $g$ be a basic operation of $\mathbf{B}$ of arity $n$. Then $\left(g h^{[n]}\right)^{\sigma} \leq g^{\sigma} h^{[n] \sigma}$ by Corollary 2.31(ii) since $h^{[n]}$ is $(\sigma, \sigma)$ continuous and $g$ is smooth. Thus, by Theorem $3.5 h^{\sigma}$ is a homomorphism and $\mathbf{B}$ has the property $(\mathrm{PH})$.

It is not hard to show that the class $\Re$ of all $\operatorname{DLS}_{\mu} \mathrm{s}$ is closed under the formation of subalgebras and homomorphic images, but it is not closed under the formation of direct products. In fact, $\mathrm{V}(\Re)$ does not have the property (PH). This follows from Example 3.13 and the obvious fact that finite DLEs are smooth.

THEOREM 3.27. If the varieties $\mathfrak{B}_{0}$ and $\mathfrak{B}_{1}$ of $\mathrm{DLE}_{\mu} s$ have the property $(\mathrm{PH})$, then so does $\mathfrak{B}_{0} \vee \mathfrak{B}_{1}$.

Proof. We need only observe that any ultraproduct of members of $\mathfrak{V}_{0} \cup \mathfrak{V}_{1}$ belongs to either $\mathfrak{B}_{0}$ or $\mathfrak{B}_{1}$.

\section{Canonical varieties}

Having seen that preservation of homomorphisms by canonical extensions is a rather common phenomenon, we now consider classes of $\mathrm{DLE}_{\mu} \mathrm{s}$ with this property, and ask which properties of the individual algebras are preserved.

Definition 4.1. A class $\mathscr{R}$ of $\operatorname{DLE}_{\mu} \mathrm{s}$ is said to be canonical if $\mathscr{R}$ has the property $(\mathrm{PH})$ and is closed under canonical extensions.

\subsection{Test algebras}

THEOREM 4.2. Suppose $\mathfrak{B}$ is a variety of DLEs with the property $(\mathrm{PH})$, and $\AA$ is a class that generates $\mathfrak{B}$. If $\mathbf{A}^{\sigma} \in \mathfrak{V}$ for all $\mathbf{A} \in \mathrm{P} u(\mathfrak{R})$, then $\mathfrak{B}$ is canonical.

Proof. First consider $\mathbf{A} \in \mathbf{P}(\Re)$. Then $\mathbf{A} \simeq \prod_{i \in I} \mathbf{A}_{i}$, with $\mathbf{A}_{i} \in \Re$ for all $i \in I$. Consequently $\mathbf{A}$ has a Boolean decomposition $h: \mathbf{A} \rightarrow \Pi_{x \in X} \mathbf{B}_{x}$, where each $\mathbf{B}_{x} \in \mathrm{P} u(\Re)$. This implies that $\mathbf{A}^{\sigma} \simeq \Pi_{x \in X} \mathbf{B}_{x}^{\sigma}$ and that each $\mathbf{B}_{x}^{\sigma} \in \mathfrak{B}$, so $\mathbf{A}^{\sigma} \in \mathbf{P}(\mathfrak{B})=\mathfrak{B}$. Next consider $\mathbf{A} \in \mathrm{SP}(\mathfrak{K})$. Then there exists an injective homomorphism $h: \mathbf{A} \rightarrow \mathbf{B} \in \mathbf{P}(\mathfrak{I})$. Now $\mathfrak{B}$ has the property $(\mathrm{PH})$, so $h^{\sigma}: \mathbf{A}^{\sigma} \rightarrow \mathbf{B}^{\sigma}$ is also an injective homomorphism. Therefore, $\mathbf{A}^{\sigma} \in \mathfrak{B}$. Finally, consider an arbitrary member $\mathbf{A}$ of $\mathfrak{B}$. Then there exists a surjective homomorphism $h: \mathbf{B} \rightarrow \mathbf{A}$ with $\mathbf{B} \in \mathrm{SP}(\Re)$. By Theorem $3.7 h^{\sigma}: \mathbf{B}^{\sigma} \rightarrow \mathbf{A}^{\sigma}$ is also a surjective homomorphism, and hence $\mathbf{A}^{\sigma} \in \mathfrak{B}$.

We digress to observe that this result throws a new light on the celebrated Fine-van Benthem-Goldblatt theorem from modal logic which, in Goldblatt's algebraic formulation states that if a class $\subseteq$ of structures is closed under 
ultraproducts, then the variety generated by the complex algebras of structures in $\widetilde{S}$ is canonical. We prove below that the class $\mathscr{\mathfrak { A }}$ consisting of the complex algebras of all the members of $\subseteq$ and the variety $\mathfrak{B}$ generated by $\mathscr{\mathfrak { I }}$ satisfy the hypothesis of the preceding theorem, and thus give a new proof of the Finevan Benthem-Goldblatt theorem. However, it would not be correct to say that we have discovered a simple proof of this important result, for our argument makes use of some difficult results from Goldblatt's paper [12].

Following Goldblatt, we denote the complex algebra of a structure $\mathbf{S}$ by $\mathbf{S}^{+}$, and let $\widetilde{\Xi}^{+}=\left\{\mathbf{S}^{+}: \mathbf{S} \in \widetilde{\subseteq}\right\}$.

THEOREM 4.3. If $\subseteq$ is a class of structures closed under ultraproducts, then the variety $\mathfrak{B}$ generated by the class $\Re=\Im^{+}$is canonical.

Proof. The variety $\mathfrak{B}$ has the property $(\mathrm{PH})$, as it is a variety of BAOs and thus of DLMs. Furthermore, for every ultraproduct $\mathbf{A}=\left(\prod_{i \infty} \mathbf{A}_{i}\right) / x=$

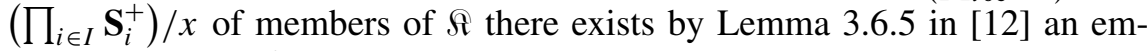
bedding $\mathbf{A} \hookrightarrow \mathbf{T}^{+}$where $\mathbf{T}$ is the ultraproduct of the structures $\mathbf{S}_{i}$, modulo the same ultrafilter $x$, Thus there is an embedding $\mathbf{A}^{\sigma} \hookrightarrow\left(\mathbf{T}^{+}\right)^{\sigma}$. By Theorem 3.6.1 in [12], $\left(\mathbf{T}^{+}\right)^{\sigma}$ can be embedded in $\mathbf{U}^{+}$for some ultrapower $\mathbf{U}_{\mu}$ of $\mathbf{T}$. Thus $\mathbf{A}^{\sigma} \in \mathfrak{B}$, Theorem 4.2 therefore applies, and $\mathfrak{B}$ is canonical.

After this digression, we note some immediate, but important consequences of Theorem 4.2.

Corollary 4.4. Suppose $\Re$ is an elementary class of DLEs with the property $(\mathrm{PH})$. If $\AA$ is canonical, then so is $\mathrm{V}(\Re)$.

COROLLARY 4.5. If $\mathfrak{B}_{1}$ and $\mathfrak{B}_{2}$ are canonical varieties, then so is $\mathfrak{B}_{1} \vee \mathfrak{V}_{2}$.

Proof. Given canonical varieties $\mathfrak{B}_{1}$ and $\mathfrak{B}_{2}$, apply Theorem 4.2 with $\mathfrak{\Re}=$ $\mathfrak{B}_{1} \cup \mathfrak{V}_{2}$, noting that $\mathfrak{V}_{1} \vee \mathfrak{V}_{2}$ has the property $(\mathrm{PH})$ by Theorem 3.27.

COROLLARY 4.6. Every finitely generated variety of DLEs is canonical.

Proof. Given a variety $\mathfrak{Y}$ generated by a finite DLE A, apply Theorem 4.2 with $\mathfrak{R}=\{\mathbf{A}\}$, noting that $\mathfrak{B}$ has the property $(\mathrm{PH})$ by Theorem 3.22.

Many of the varieties of DLEs that arise as the semantic equivalents of propositional logics are finitely generated and, as H. Priestley has pointed out to us, so are the varieties mainly studied in natural duality theory. This result therefore has many potential applications that have yet to be explored.

Corollary 4.7. Suppose $\mathfrak{B}$ is a variety of DLEs with (PH), and suppose $\mathrm{Si}(\Re)$ is an elementary class. If $\mathrm{Si}(\Re)$ is canonical, then so is $\mathfrak{B}$. 
For a simple application of this corollary, consider the variety $\mathfrak{B}$ of all pseudocomplemented distributive lattices. (See [1].) The subdirectly irreducible members are the algebras $\mathbf{B} \oplus \mathbf{1}$, consisting of a Boolean algebra $\mathbf{B}$, with a new top element added. Hence $\mathrm{Si}(\mathfrak{B})$ is an elementary class. Also, $(\mathbf{B} \oplus \mathbf{1})^{\sigma}=\mathbf{B}^{\sigma} \oplus \mathbf{1}$, and $\operatorname{Si}(\mathfrak{B})$ is therefore canonical. Hence $\mathfrak{B}$ is canonical. Incidentally, all the proper subvarieties of $\mathfrak{B}$ are also canonical, for each of them is generated by a single finite algebra $\mathbf{B} \oplus \mathbf{1}$.

\subsection{Stable, expanding, and contracting terms}

An obvious way to try to prove that an identity $s \approx t$ is preserved by canonical extensions is to attempt to show that $s^{\mathbf{A}^{\sigma}}=\left(s^{\mathbf{A}}\right)^{\sigma}$ and $t^{\mathbf{A}^{\sigma}}=\left(t^{\mathbf{A}}\right)^{\sigma}$. If these equalities hold, then we say that the terms $s$ and $t$ are stable on the algebra $\mathbf{A}$, or on the class of algebras A. Similarly, to prove that an inequality $s \leq t$ is preserved, it suffices to show that $s^{\mathbf{A}^{\sigma}} \leq\left(s^{\mathbf{A}}\right)^{\sigma}$ and $t^{\mathbf{A}^{\sigma}} \geq\left(t^{\mathbf{A}}\right)^{\sigma}$. If these inequalities hold, then we say that $s$ is contracting and that $t$ is expanding. The property of stability played an essential role in [15], although it was not explicitly mentioned there. All three properties were used in [13].

We now have a new way of showing that certain terms have these properties.

Theorem 4.8. Suppose $\mathbf{A}$ is a $\mathrm{DLE}_{\mu}$ and $t$ is a $\mu$-term,

(i) If, for every operation symbol $\omega$ that occurs in $t, \omega^{\mathbf{A}^{\sigma}}$ is $\left(\iota^{\uparrow}, \iota^{\uparrow}\right)$-continuous, then t is contracting on $\mathbf{A}$.

(ii) If, for every operation symbol $\omega$ that occurs in $t, \omega^{\mathbf{A}}$ is isotone, then $t$ is expanding on $\mathbf{A}$.

(iii) If, for every operation symbol $\omega$ that occurs in $t, \omega^{\mathbf{A}^{\sigma}}$ is $(\iota, \iota)$-continuous, then $t$ is stable on $\mathbf{A}$.

Proof. (i) From the fact that continuity is preserved by composition we infer that $t^{\mathbf{A}^{\sigma}}$ is $\left(\iota^{\uparrow}, \iota^{\uparrow}\right)$-continuous. In particular, $t^{\mathbf{A}}$ is $\left(\sigma, \iota^{\uparrow}\right)$-continuous. Hence $t^{\mathbf{A}^{\sigma}} \leq\left(t^{\mathbf{A}}\right)$ by Theorem $2.15, t$ is contracting on $\mathbf{A}$.

(ii) By Theorem 2.33.

(iii) In this case, $t^{\mathbf{A}}$ is $(i, i)$-continuous, and it is therefore both $\left(\sigma, \iota^{\uparrow}\right)$ continuous and $\left(\sigma, l^{\downarrow}\right)$-continuous. We conclude as before that $t$ is contracting on $\mathbf{A}$. Also, by Theorem $2.15, t^{\mathbf{A}^{\sigma}} \geq t^{\mathbf{A}^{\pi}}=\left(t^{\mathbf{A}}\right)^{\pi} \geq\left(t^{\mathbf{A}}\right)^{\sigma}$, so $t$ is expanding on $\mathbf{A}$. Therefore $t$ is stable on $\mathbf{A}$.

These observations provide a surprisingly powerful tool for proving canonicity. Two simple examples follow. The first example is the principal theorem of [8].

THEOREM 4.9. Every variety of DLOs is canonical. 
Proof. In any DLO A, all the basic operations $\omega^{\mathbf{A}}$ are of course isotone, and by Theorem $2.23, \omega^{\mathbf{A}^{\sigma}}$ is $\left(\iota^{\uparrow}, \iota^{\uparrow}\right)$-continuous. Hence, by the preceding theorem, every term is stable on $\mathbf{A}$.

An Ockham algebra is a DLE $\mathbf{A}=\left(\mathbf{A}_{0}, f\right)$ with $f$ a lattice anti-endomorphism. Observe that $f$ is not required to take 0 into 1 and 1 into 0 . Since lattice isomorphisms are $(i, i)$-continuous, so are lattice anti-endomorphisms. Hence every variety of Ockham algebras is canonical. This can be generalized by considering DLEs with several unary operations, each of which is either a lattice endomorphism or a lattice anti-endomorphism. Let us call such algebras generalized Ockham algebras.

THEOREM 4.10. Every variety of generalized Ockham algebras is canonical.

\subsection{The Chang $M V$-algebra}

In [17], R. Kramer and R. Maddux give a particularly striking example of the failure of canonicity, a relation algebra $\mathbf{A}$ with the property that no complete extension of $\mathbf{A}$ is in the variety generated by $\mathbf{A}$. We will show that the wellknown Chang MV-algebra also has this property. This is a stronger version of a result in [10] which states that the canonical extension of the Chang MValgebra is not an MV-algegbra.

Information about MV-algebras in general and about the Chang MV-algebra in particular can be found e.g. in [10], but actually the facts that we need are few and simple. MV-algebras are DLEs with a binary operation $\rightarrow$ that is isotone in its second argument, with $x \rightarrow y=1$ whenever $x \leq y$. They also satisfy the identity

$$
(x \rightarrow y) \rightarrow y=x \vee y .
$$

The only fact about the Chang MV-algebra that will be used is that it contains an infinite descending sequence $1=a_{0}>a_{1}>\cdots$ with $a_{m} \rightarrow a_{n}=a_{n \ominus m}$ for all $m, n \in \omega$, where $\ominus$ is trunkated subtraction.

Assuming now that the Chang algebra has a complete MV-extension, let $a_{\infty}=\bigwedge_{n \in \omega} a_{n}$. For a fixed $m \in \omega$ we have $a_{m} \rightarrow a_{\infty} \leq a_{n \ominus m}$ for all $n \in \omega$, and hence $a_{m} \rightarrow a_{\infty} \leq a_{\infty}$. Consequently

$$
\left(a_{m} \rightarrow a_{\infty}\right) \rightarrow a_{\infty}=1
$$

while $a_{m} \vee a_{\infty}=a_{m}<1$ for $m>0$. This contradicts our assumption that the extension is an MV-algebra. 


\section{Epilogue}

The broader definition of the notion of a canonical extension introduced in this paper makes this concept available for the study of many interesting classes of algebras that did not fall within the scope of the earlier definitions. Rather than investigating individual classes in detail, we have tried to develop techniques that apply in many different settings. The fact that the broader concepts have not resulted in a weaker or a more complex theory, but have instead led to powerful new techniques, suggests that we are on the right track.

Looking briefly at what might be ahead, we will also focus on questions of general nature. There will be still further generalizations of the notion of a canonical extension. The most obvious one is that the requirement of boundedness will be dropped. In fact, many potential applications involve residuated lattices, and in many cases these are not bounded. Other possible generalizations consist in taking more general underlying posets. The lattice ordered case has already been investigated some, see [7], but the topologies, for instance, have not been investigated in this setting.

We may wonder whether the present notion of canonical extension is "the right one" or "the best one". This is an imprecise question that does not possess a definitive answer. In the case of DLs, there is a rather convincing interpretation supporting the present definition: Think of the universe $A$ of a DL A as a set of properties, closed under finite disjunction and conjunction, and of the universe $A^{\sigma}$, of $\mathbf{A}^{\sigma}$ as the closure of $A$ under infinitary disjunctions and conjunction, In the presence of auxiliary operations $f=\omega^{\mathbf{A}}$, the situation is less clear. If we think of $f$ as a new logical connective, then $f^{\sigma}$ and $f^{\pi}$ are different infinitary connectives. Which one should we use, and why? We have mostly worked with $f^{\sigma}$, noting that any result we obtained could be dualized to provide a corresponding result for $f^{\pi}$. However, canonicity and dual canonicity are not equivalent. E.g., the variety of all Heyting algebras is not canonical, but it is dually canonical. Of course this means that the variety of all dual Heyting algebras is canonical. There are other cases where, in order to stay within a given variety, one must use "mixed" extensions, taking the canonical extensions of some of the basic operations and the dual canonical extensions of others. Even worse, in some cases both extensions must be used (at different spots in the equations defining the variety) in order to ensure canonicity. This shows that our present notion can sometimes be improved upon, but there are situations where no choice within the given type setting is "right". 


\section{REFERENCES}

1. Balbes, R., and Dwinger, Ph., Distributive Lattices, Missouri University Press, 1974.

2. Banaschewski, B., Coherent frames, in Proceedings of the Conference on Topological and Categorical Aspects of Continuous Lattices (Workshop IV, 1979). Edited by B. Banaschewski and R. E. Hoffmann. Lecture Notes in Math. 871 (1981), 1-11.

3. Birkhoff, G., Lattice Theory, fourth edition, Amer. Math. Soc. Coll. Publ. vol. 25, 1967.

4. Burris, S., and Sankappanavar, H. P., A Course in Universal Algebra, Graduate Texts in Math., vol. 78, 1981.

5. Engelking, R., General Topology, Polish Scientific Publishers, Warsaw, 1977.

6. Gehrke, M., The order structure of Stone spaces and the $T_{D}$-separation axiom, Z. Math. Logik Grundlag. Math. 37 (1991), 5-15.

7. Gehrke, M., and Harding, J., Bounded Lattice Expansions, J. Algebra 238 (2001), 345-371.

8. Gehrke, M., and Jónsson, B., Bounded distributive lattices with operators, Math. Japon. 40 (1994), 207-215.

9. Gehrke, M., and Jónsson, B., Monotone bounded distributive lattice expansions, Math. Japon. 52 (2000), 197-213.

10. Gehrke, M., and Priestley, H. A., Non-canonicity of MV-algebras, Houston J. Math. 28 (2002), 449-455.

11. Gierz, G., Hofmann K. H., Keimel, K., Lawson, J. D., Mislove, M., and Scott, D. S., A Compendium of Continuous Lattices, Springer-Verlag, 1980.

12. Goldblatt, R., Varieties of complex algebras, Ann. Pure Appl. Logic 44 (1989), 173-242.

13. Jónsson, B., On the canonicity of Sahlqvist identities, Studia Logica 53 (1994), 473-491.

14. Jónsson, B., Congruence distributive varieties, Math. Japon. 42 (1995), 353-401.

15. Jónsson, B., and Tarski, A., Boolean algebras with operators, I, Amer. J. Math. 73 (1951), 891-939.

16. Jónsson, B., and Tarski, A., Boolean algebras with operators, II, Amer. J. Math. 74 (1952), $127-162$.

17. Kramer, R., and Maddux, R., Equations not preserved by complete extensions, Algebra Universalis 15 (1982), 86-89.

18. Priestley, H. A., Representations of distributive lattices by means of ordered topological spaces, Bull. London Math. Soc. 2 (1970), 186-190.

19. Priestley, H. A., Intrinsic spectral topologies, Papers on General Topology and Applications (Flushing, NY, 1992), 78-95, Ann. New York Acad. Sci. 728 (1994).

20. Priestley, H. A., Varieties of distributive lattices with unary operations, I, J. Austral. Math. Soc. 63 (1997), 165-207.

21. Ribeiro, H., A remark on Boolean algebras with operators, Amer. J. Math. 74, 163-167.

22. Sofronie-Stokkermans, V., Duality and canonical extensions of bounded distributive lattices with operators, and applications to the semantics of non-classical logics, I, II, Studia Logica 64 (1999), 93-132 and 151-172.

23. Stone, M. H., Topological representations of distributive lattices and Browerian logics, Casopis pest. Mat. 67 (1937), 1-25.

DEPARTMENT OF MATHEMATICAL SCIENCES

NEW MEXICO STATE UNIVERSITY

P.O. BOX 30001

LAS CRUCES, NM 88003-8001

USA

E-mail:mgehrke@nmsu.edu
DEPARTMENT OF MATHEMATICS

VANDERBILT UNIVERSITY

NASHVILLE, TENNESSEE 37235

USA

E-mail: jonsson@math.vanderbilt.edu 